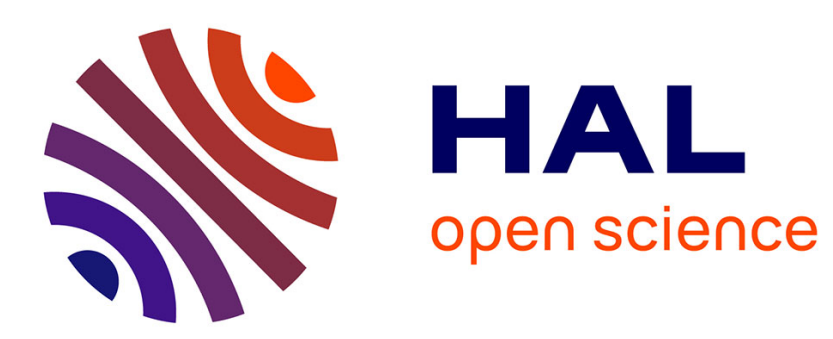

\title{
A set of enhanced formulations for existing nonlinear homogenization schemes and their evaluation
}

\author{
Amna Rekik, François Auslender, Michel Bornert
}

\section{To cite this version:}

Amna Rekik, François Auslender, Michel Bornert. A set of enhanced formulations for existing nonlinear homogenization schemes and their evaluation. European Journal of Mechanics - A/Solids, 2015, pp.10.1016/j.euromechsol.2014.10.001. 10.1016/j.euromechsol.2014.10.001 . hal-01071907

\section{HAL Id: hal-01071907 https://hal.science/hal-01071907}

Submitted on 7 Oct 2014

HAL is a multi-disciplinary open access archive for the deposit and dissemination of scientific research documents, whether they are published or not. The documents may come from teaching and research institutions in France or abroad, or from public or private research centers.
L'archive ouverte pluridisciplinaire HAL, est destinée au dépôt et à la diffusion de documents scientifiques de niveau recherche, publiés ou non, émanant des établissements d'enseignement et de recherche français ou étrangers, des laboratoires publics ou privés. 


\title{
A set of enhanced formulations for existing nonlinear homogenization schemes and their evaluation
}

\author{
Amna Rekik ${ }^{1}$, François Auslender ${ }^{2}$, Michel Bornert $^{3}$ \\ 1 Univ. Orléans, INSA-CVL, PRISME, EA4229, 45072 Orléans, France \\ 2 Université Blaise Pascal, Institut Pascal, UMR CNRS 6602, BP 10448, 63000 Clermont-Ferrand, France \\ ${ }^{3}$ Université Paris Est, Laboratoire Navier (ENPC/IFSTTAR/CNRS UMR 8205), \\ ENPC, 6 et 8 avenue Blaise Pascal, 77455 Marne la Vallée Cedex, France
}

\begin{abstract}
On the basis of non-biased comparative evaluations of various linearization procedures used in nonlinear homogenization, performed both at the global and local scales for power-law composites [37, 39, 40], we propose in this paper six ad hoc enhancements of some of the linearization procedures considered in [39]. Both "stress-strain" approaches (the secant and affine formulations) or "variational formulations" (the tangent second-order method [33]) are considered. The main idea consists in proposing alternatives for the usual reference strains used by the secant, affine and tangent second-order procedures. The new linear comparison composites generated by the linearization step around the chosen alternative descriptors of the strain field statistics explicitly account for either intraphase strain fluctuations or both inter- and intraphase strain fluctuations. As a first illustration, the relevance and limitations of the enhanced linearization procedures are tested for rigidly-reinforced and porous power-law composites. For isochoric loadings, it is shown that two variants of the enhanced tangent second-order formulation lead to accurate estimates of the exact effective response which are in good agreement with the efficient second-order scheme of Ponte Castañeda [35]. Further, the modified secant formulation provides good results for strongly nonlinear rigidly-reinforced composites away from low particulate volume fraction and the percolation threshold; however some new inherent limitations of secant formulations are also established. At last, a very discriminant situation is tested: it consists of a porous medium submitted to a pure hydrostatic loading at low pore concentrations. It is shown that one variant of the proposed enhanced second-order formulations leads to accurate estimates alike the efficient and more sophisticated formulations proposed in [3, 9].
\end{abstract}

Key words: Nonlinear homogenization; Linearization; Secant; Affine; Variational procedures; Local field fluctuations; Reinforced composites; Porous media

\section{Introduction}

Nonlinear homogenization techniques are powerful methods allowing the derivation of bounds or estimates for the effective properties of heterogeneous nonlinear composites from both their local constitutive laws and the statistical description of their microstructure. These techniques rely on two steps: the linearization and the linear homogenization. The first step, through the linearization of the phase constitutive relations defines a linear comparison composite (LCC) whose microstructure is in general taken to be similar to that of the nonlinear composite. In the literature, several expressions of the corresponding local linearized behaviour have been proposed. For the secant method [2], the phases of the LCC are defined from the secant moduli $(9)_{1}$ of the actual phases. For the affine formulation due to Masson and Zaoui [24], thermoelastic comparison 
materials are defined from tangent operators $(9)_{2}$ of the phases. The classical secant and affine estimates are evaluated at the first-moment of the strain field over the phases and yield too stiff responses [10, 39]. To improve the affine formulation, Chaboche et al. [8] proposed to replace the tangent anisotropic operator by a softer isotropic simplification. Simultaneously, Brenner et al.[6] proposed a "modified affine" formulation that accounts for the intraphase heterogeneity, by analogy to the "modified secant" approach proposed by Suquet in [42] for the special case of power-law materials. The latter is equivalent to the variational approach developed by Ponte Castañeda [32] which consists in the use of an optimally chosen LCC with reference to a variational formulation. It delivers bounds exact to the first-order in the heterogeneities contrast. The same author proposed in [33] an alternative approach relying on a second-order expansion of the strain energy of a constitutive phase around a reference strain taken as the strain average over this phase. This model yields estimates that are exact to the second-order in the contrast but which can violate the variational bounds in some special cases, as near the percolation phenomenon [23]. For this reason Ponte Castañeda [35] proposed an improved second order method that makes use of a generalized secant moduli incorporating both interand intra-phase strain fluctuations. However, one of the reference strain used in this new procedure is not fully defined by stationary conditions relying on the nonlinear composite and thus induces some limitations for the second order variant proposed in [35]. Accordingly, Idiart et al. [14, 16] introduced an alternative prescription for this reference strain in a new and third variant of the second-order method which provides slightly improved estimates for the effective and local responses in comparison with the earlier prescription [35]. The new prescription for the reference strain field does still not ensure fully stationarity conditions. It is noteworthy that the second-order methods do not directly deliver an effective stress-strain relation but rather an effective strain-energy which needs to be differentiated. Moreover, the affine formulation is less accurate compared with the second-order methods. In particular, the affine scheme is not exact to second order in the contrast and, more critically, is not associated with an overall potential. To overcome these difficulties, Lahellec and Suquet [21] proposed a new approximate scheme which closes the gap between the affine and the second order procedure and gives results exact to second order in the contrast.

As seen above, numerous linearization methods are available and their relative merits are not easy to understand to an unexperienced user. However, they have been accurately evaluated by different authors - e.g., Moulinec and Suquet [29], Rekik et al. [37, 39, 40], Lahellec and Suquet [21] and Idiart et al. [18] - without any ambiguity related to the approximations induced by the linear homogenization estimates of the LCC. In particular, Rekik et al. in [37, 39] proposed a methodology allowing the evaluation of the sole effect of linearization schemes without the classical bias present in earlier evaluations such as the use of linear homogenization schemes available in the literature for assessing the LCC behavior. It relies on an exact treatment of both the nonlinear and linear homogenization problems using the finite element method. In $[38,39,40]$, various nonlinear homogenization schemes such as the classical secant scheme (referred to as SEC), the variational procedure (VAR), the original affine formulation (AFF-ANI) and its isotropic simplification (AFF-ISOT), the original (SOE-1) and improved (SOE-2) second-order procedures as well as the Lahellec and Suquet (LS) formulation were compared with regard to their predictions in terms of overall responses and local field statistics for the special case of power-law two-phase composites with different contrast between the phases ranging from the rigidly-reinforced composites to porous materials. Based on the main results of these comparisons, we propose in this paper some improvements for existent "stressstrain" approaches (SEC, AFF-ANI) or "potential-based" approaches such as the variational (VAR) and tangent second-order (SOE-1) formulations. The main idea consists in evaluating the secant shear modulus $\mu_{s c t}^{r}\left(\varepsilon^{r}\right)$ of the secant $(9)_{1}$ or tangent $(9)_{2}$ stiffness tensor at a reference strain $\hat{\varepsilon}_{e q}^{r}(14)_{1}$ incorporating both the inter- and intra-phase strain fluctuations. Such an incorporation of both the inter- and intra-phase strain fluctuations is applied to the SEC, AFF-ANI and SOE-1 approaches and aims to soften the secant and tangent operators associated with these approaches and thus to improve them since these procedures have 
been shown in $[39,40]$ to provide too stiff estimates of the effective behavior. For the affine formulations (AFF-ANI and AFF-ISOT), it is also proposed to make use of a shear modulus which is intermediate between the standard secant $\mu_{s c t}^{r}\left(\bar{\varepsilon}^{r}\right)$ and tangent $\mu_{t g t}^{r}\left(\bar{\varepsilon}^{r}\right)$ ones for the perpendicular direction $F^{r}$ of the tangent operator (30). Such a linearization aims to improve the classical and simplified affine formulations especially for strong nonlinearities since numerical comparisons performed in [39] have shown that the exact solution lies between the AFF-ANI and AFF-ISOT responses. At last, for the tangent second-order procedure, we also propose to soften the secant shear modulus $\mu_{s c t}^{r}\left(\bar{\varepsilon}_{e q}^{r}\right)$ (resp. the tangent shear modulus $\mu_{\text {tgt }}^{r}\left(\bar{\varepsilon}_{e q}^{r}\right)$ ) associated with the orthogonal direction $F^{r}$ (resp. the parallel direction $E^{r}$ ) of the tangent operator by evaluating it at the second-order moment of the strain field in each phase $\overline{\bar{\varepsilon}}_{\text {eq }}^{r}(13)_{1}$ or at the strain descriptor $\hat{\varepsilon}_{e q}^{r}(14)_{1}$.

Then, in order to evaluate the efficiency of these proposed enhanced linearization schemes, we present a first comparative and non-biased study at the macroscopic scale with some of the most used or famous linearization procedures, i.e. the secant, affine, second-order formulations as well as the Lahellec and Suquet procedure. For that, we focus our attention on rigidly-reinforced and porous power-law materials. These materials are of extreme heterogeneity in the contrast and therefore constitute discriminating cases to study.

The structure of the paper is as follows. In Section 2, we briefly recall the various linearization schemes which will be used in the sequel either to design new linearization procedures or for comparative purposes. In Section 3, relying on some relevant observations and conclusions derived from non-biased comparative evaluations of the linearization schemes presented in Section 2, new and enhanced formulations are proposed. A first evaluation of their performances at the macroscopic scale is carried out in Section 4 where the new proposed linearization schemes are compared with some of the most used linearization procedures for nonlinear two-phase composites made of identical spherical inclusions - either pores or quasi-incompressible isotropic linear elastic particles - embedded in a nonlinear isotropic matrix following a Ramberg-Osgood law. Conclusions are summarized in Section 5.

The tensor notation used herein is a fairly standard one. Products containing dots denote summation over repeated indices. For example, $L: \varepsilon=L_{i j k l} \varepsilon_{k l} e_{i} \otimes e_{j}$ and $E:: F=E_{i j k l} F_{k l i j}$ where $e_{i}(i=1,2,3)$ is a time-independent orthogonal cartesian basis and the operation $\otimes$ denotes the classical tensorial diadic product.

2. Reminder of general principles of nonlinear homogenization schemes and their evaluation by a non-biased methodology

\subsection{Nonlinear effective properties}

The main objective of homogenization is to predict the macroscopic behaviour of composite materials in terms of the behaviour of their constituents and prescribed statistical information about their microstructure. In this framework, we consider composite materials made of $N$ different homogeneous constituents, each occupying a volume $V_{r}:(r=1, \ldots, N)$, "periodically or randomly" spatially distributed in a specimen occupying a volume $V=\bigcup_{r=1}^{N} V_{r}$, and submitted to mechanical loadings which are assumed to be macrohomogenous [13], thus making the scale transition possible. The constitutive behaviour of each phase is characterized by a convex single potential or strain energy function $w^{r}$, such that the stress $\sigma$ and strain $\varepsilon$ tensors are related by

$$
\sigma=\frac{\partial w(x, \varepsilon)}{\partial \varepsilon}, \quad w(x, \varepsilon)=\sum_{r=1}^{N} \chi^{r}(x) w^{r}(\varepsilon),
$$


where $\frac{\partial}{\partial \varepsilon}$ denotes differentiation with respect to $\varepsilon$, and the characteristic functions $\chi^{r}(x)$ serve to describe the microstructure, set to 1 if the position vector $x$ is in phase $r$, and 0 otherwise. This constitutive relation corresponds to a nonlinear elastic behavior within the context of small strain. Let $\langle$.$\rangle and \langle\text {. }\rangle^{r}$ denote the volume averages over the composite and over phase $r$, respectively. Under the assumed condition of macrohomogeneity $\langle\sigma: \varepsilon\rangle=\langle\sigma\rangle:\langle\varepsilon\rangle$, the effective behaviour of the composite, which is defined as the relation between the average stress $\bar{\sigma}=\langle\sigma\rangle$ and the average strain $\bar{\varepsilon}=<\varepsilon>$ can be characterized by an effective strain potential $\tilde{w}$, such that

$$
\bar{\sigma}=\frac{\partial \tilde{w}(\bar{\varepsilon})}{\partial \bar{\varepsilon}}, \quad \tilde{w}(\bar{\varepsilon})=\inf _{u \in \kappa(\bar{\varepsilon})}\langle w(x, \varepsilon(u))\rangle,
$$

where $\kappa(\bar{\varepsilon})=\left\{u\right.$ continuous, $\varepsilon(u)=\frac{1}{2}\left(\nabla u+{ }^{T} \nabla u\right)$ and $\langle\varepsilon(u)>=\bar{\varepsilon}\}$. The local strain field $\varepsilon(u)$ solution of the variational theorem $(2)_{2}$ is also solution of the so-called nonlinear local problem consisting of the following set of equations

$$
\left\{\begin{array}{l}
\operatorname{div}(\sigma)=0, \quad \forall x \in V \\
\sigma(x)=\sum_{r=1}^{N} \chi^{r}(x) g^{r}(\varepsilon(x)), \quad \forall x \in V \\
\varepsilon(u(x))=\frac{1}{2}\left(\nabla u(x)+{ }^{t} \nabla u(x)\right), \quad \forall x \in V \\
<\varepsilon(u)>=\bar{\varepsilon},
\end{array}\right.
$$

where $g^{r}(\varepsilon)=\frac{\partial w^{r}(\varepsilon)}{\partial \varepsilon}$ denotes the constitutive law of phase $r$.

Under the assumed condition of macrohomogeneity, the average stress $\bar{\sigma}$ might be equivalently calculated either by means of the effective potential $\tilde{w}(\bar{\varepsilon})$ through Eq. $(2)_{1}$ or by averaging the local stress field over the RVE, i.e.

$$
\bar{\sigma}=\langle\sigma\rangle .
$$

Therefore, the derivation of the effective behavior by the potential approach (2) or by the stress-strain approach (3), (4) are equivalent.

\subsection{Nonlinear homogenization: general principles}

The nonlinear problem, defined by Eqs. (3) and (4), can be approached using nonlinear homogenization theories based on two steps: linearization of the constitutive behavior of each phase around some reference strains and linear homogenization of the resulting linear comparison composite.

Through the first step, which consists in linearizing the constitutive behavior, the nonlinear local problem (3) is approached by the following system

$$
\left\{\begin{array}{l}
\operatorname{div}(\sigma)=0, \quad \forall x \in V \\
\sigma(x)=\sum_{r=1}^{N} \chi^{r}(x)\left(L^{r}: \varepsilon(x)+\tau^{r}\right), \quad \forall x \in V \\
\varepsilon(u(x))=\frac{1}{2}\left(\nabla u(x)+{ }^{t} \nabla u(x)\right), \quad \forall x \in V \\
<\varepsilon(u)>\bar{\varepsilon}, \\
L^{r}=L^{r}\{\varepsilon(x), x \in V\}, \tau^{r}=\tau^{r}\{\varepsilon(x), x \in V\},
\end{array}\right\} \quad \begin{aligned}
& \\
& \text { nonlinear relations }
\end{aligned}
$$

where $L^{r}\{\varepsilon(x), x \in V\}$ and $\tau^{r}\{\varepsilon(x), x \in V\}$ are chosen analytical functionals of the local strain field $\varepsilon(x)$ of the local linear problem $(5)_{1}$. This problem coincides with the so-called local problem associated with a fictitious linear composite usually called the Linear Comparison Composite (LCC) [32]. The behavior of each of its individual constituents is linear thermo-elastic and defined by $\sigma=L^{r}: \varepsilon+\tau^{r}$.

The second step, which consists of the linear homogenization stage, aims at solving the local linear problem $(5)_{1}$ and deriving its effective behavior by means of linear homogenization techniques. At this stage, it should 
be emphasized that the effective behavior associated with the approximate system (5) might be computed from two different ways. For linearization procedures that will be referred to in the sequel as "stress-strain approaches", the macroscopic stress is evaluated as the average value of the local stress field within the LCC, i.e. $\bar{\sigma}=\langle\sigma\rangle$ with $\sigma(x)$ solution of the local linear problem (5) $)_{1}$. For linearization procedures called in what follows "potential-based approaches", the effective stress is defined according to Hill's theorem $(2)_{1}$ with an effective potential $\tilde{w}(\bar{\varepsilon})=\sum_{r=1}^{N} f^{r}<w^{r}(\varepsilon)>^{r}$ approximated by

$$
\tilde{w}(\bar{\varepsilon}) \simeq \sum_{r=1}^{N} f^{r} w_{a p p}^{r}, \quad w_{a p p}^{r}=w_{a p p}^{r}\{\varepsilon(x), x \in V\}
$$

where $f^{r}=<\chi^{r}>$ denotes the volume fraction of phase $r$ and the quantities $w_{a p p}^{r}\{\varepsilon(x), x \in V\}$, similarly to $L^{r}\{\varepsilon(x), x \in V\}$, are chosen analytical functionals of the local strain field $\varepsilon(x)$ of the local linear problem $(5)_{1}$, consistent with the linearization stage. Note that for potential-based approaches the linearization of the constitutive behavior yields two different types of relations. The first one defines the LCC constitutive behavior through Eq. $(5)_{2}$ while the second defines the approximated per-phase energy $w_{a p p}^{r}$ as a nonlinear analytical functional of the local strain field of the local linear problem (see Eq. (6) 2 ). Illustrations of the linearization stage for potential-based approaches are given in Section B.2 for the tangent SOE-1 and improved SOE-2 second-order procedures as well as for the LS formulation.

Although the considered linearization schemes and the methodology developed here could be applied to more general situations, this work is restricted, for the sake of clarity, to composites made of isotropic constitutive phases, with a strain energy potential of the form

$$
\omega(\varepsilon)=\frac{9}{2} k\left(\varepsilon_{m}\right)^{2}+w_{d e v}\left(\varepsilon_{e q}\right)
$$

where $\varepsilon_{m}=(i: \varepsilon) / 3$ and $\varepsilon_{e q}=\sqrt{\frac{2}{3} \varepsilon_{d}: \varepsilon_{d}}$ denote the isotropic part and the von Mises measure of the deviatoric part $\varepsilon_{d}$ of the strain tensor, respectively. Tensor $i$ is the second-order identity tensor while $w_{\text {dev }}\left(\varepsilon_{e q}\right)$ is a nonlinear scalar function of its argument. Accordingly, the local constitutive behavior reads

$$
\sigma(\varepsilon)=\sigma_{m} i+\frac{2}{3} \sigma_{e q} \hat{e}(\varepsilon) \quad \text { with } \quad \sigma_{m}=3 k \varepsilon_{m}, \quad \sigma_{e q}=\frac{\partial \omega_{\text {dev }}\left(\varepsilon_{e q}\right)}{\partial \varepsilon_{e q}}, \quad \hat{e}(\varepsilon)=\frac{\varepsilon_{d}}{\varepsilon_{e q}}
$$

where $\sigma_{m}=\sigma: i / 3$ and $\sigma_{e q}=\sqrt{\frac{3}{2} \sigma_{d}: \sigma_{d}}$ are the isotropic part and the von Mises measure of the deviatoric part $\sigma_{d}$ of the stress tensor. The particular loading conditions, microstructure and form of the nonlinear relation $\sigma_{e q}=g\left(\varepsilon_{e q}\right)$ considered in the numerical simulations will be described in section 2.4. For isotropic phases associated with strain energy potential $(7)$, the secant $L_{s c t}(\varepsilon)$ and tangent $L_{t g t}(\varepsilon)$ stiffness tensors are defined by

$$
\begin{aligned}
& L_{s c t}(\varepsilon)=3 k J+2 \mu_{s c t}\left(\varepsilon_{e q}\right) K \quad \text { with } \quad \mu_{s c t}\left(\varepsilon_{e q}\right)=\frac{\sigma_{e q}\left(\varepsilon_{e q}\right)}{3 \varepsilon_{e q}} \\
& L_{t g t}(\varepsilon)=\frac{\partial^{2} w}{\partial \varepsilon^{2}}(\varepsilon)=3 k J+2 \mu_{t g t}\left(\varepsilon_{e q}\right) E+2 \mu_{s c t}\left(\varepsilon_{e q}\right) F \quad \text { with } \quad \mu_{t g t}\left(\varepsilon_{e q}\right)=\frac{d \sigma_{e q}\left(\varepsilon_{e q}\right)}{3 d \varepsilon_{e q}}
\end{aligned}
$$

where $\mu_{s c t}\left(\varepsilon_{e q}\right)$ and $\mu_{t g t}\left(\varepsilon_{e q}\right)$ are the secant and tangent shear moduli, respectively. Fourth-order tensors $K=I-J$ and $J=\frac{1}{3} i \otimes i$ are the usual projectors on the subspaces of purely spherical or deviatoric second-order tensors. The tensor $I$ is the fourth-order symmetric identity tensor while fourth order tensors $E(\varepsilon)$ and $F(\varepsilon)$, defined respectively by

$$
E(\varepsilon)=\frac{2}{3} \hat{e}(\varepsilon) \otimes \hat{e}(\varepsilon), \quad F(\varepsilon)=K-E(\varepsilon),
$$


are projectors which contract the deviatoric part of a second-order tensor respectively onto the parallel $\hat{e}(\varepsilon)$ and orthogonal directions of the deviatoric strain $\varepsilon_{d}$.

\subsection{Field fluctuations and descriptors}

The inter- and intra-phase strain fluctuations are respectively described by both the per-phase strain averages and per-phase strain covariance tensors defined by

$$
\bar{\varepsilon}^{r}=<\varepsilon>^{r}, \quad C_{\varepsilon}^{r}=\left\langle\left(\varepsilon-\bar{\varepsilon}^{r}\right) \otimes\left(\varepsilon-\bar{\varepsilon}^{r}\right)\right\rangle^{r} .
$$

For composite with isotropic constitutive phases, four descriptors, usually denoted $\bar{\varepsilon}^{r}, \overline{\bar{\varepsilon}}_{e q}^{r}, \hat{\varepsilon}_{\|}^{r}, \hat{\varepsilon}_{\perp}^{r}$ are often used in mean-field nonlinear homogenization theories to describe the statistics of the local strain field. The first two descriptors $\bar{\varepsilon}^{r}, \overline{\bar{\varepsilon}}_{e q}^{r}=\sqrt{<\varepsilon_{e q}^{2}>^{r}}$ are respectively the first and second-order moments of the strain field in phase $r$. The other two descriptors, derived from both the per-phase strain averages and covariance strain tensors, account for the intra- (and inter-) phase strain fluctuations. They are defined by

$$
\hat{\varepsilon}_{\|}^{r}=\bar{\varepsilon}_{e q}^{r}+\sqrt{\frac{2}{3} E^{r}:: C_{\varepsilon}^{r}}, \quad \hat{\varepsilon}_{\perp}^{r}=\sqrt{\frac{2}{3} F^{r}:: C_{\varepsilon}^{r}}
$$

with $E^{r}=E\left(\bar{\varepsilon}^{r}\right), F^{r}=K-E^{r}$. Note that the second-order moment of the strain field $\bar{\varepsilon}_{e q}^{r}$ in phase $r$ is a function of the other three descriptors since

$$
\bar{\varepsilon}_{e q}^{r}=\sqrt{\frac{2}{3}<\varepsilon \otimes \varepsilon>^{r}:: K}=\sqrt{\left(\bar{\varepsilon}_{e q}^{r}\right)^{2}+\left(\hat{\varepsilon}_{\|}^{r}-\bar{\varepsilon}_{e q}^{r}\right)^{2}+\left(\hat{\varepsilon}_{\perp}^{r}\right)^{2}}
$$

and therefore also accounts for both the inter- and intra-phase strain fluctuations.

From these strain descriptors, we also define the following quantities which will be used hereafter

$$
\hat{\varepsilon}_{e q}^{r}=\sqrt{\left(\hat{\varepsilon}_{\|}^{r}\right)^{2}+\left(\hat{\varepsilon}_{\perp}^{r}\right)^{2}}, \quad \delta_{\|}^{r}(\varepsilon)=\hat{\varepsilon}_{\|}^{r}-\bar{\varepsilon}_{e q}^{r}=\sqrt{\frac{2}{3} E^{r}:: C_{\varepsilon}^{r}}, \quad \delta_{\perp}^{r}(\varepsilon)=\hat{\varepsilon}_{\perp}^{r}=\sqrt{\frac{2}{3} F^{r}:: C_{\varepsilon}^{r}},
$$

where $\delta_{\|}^{r}(\varepsilon)$ and $\delta_{\perp}^{r}(\varepsilon)$ denote the parallel and perpendicular measures of the fluctuations of the strain field in phase $r$, respectively. The measures of the inter and intra-phase stress fluctuations can be defined similarly as in Appendix (A). The four descriptors $\bar{\varepsilon}^{r}, \bar{\varepsilon}_{e q}^{r}, \hat{\varepsilon}_{\|}^{r}, \hat{\varepsilon}_{\perp}^{r}$ provide very useful informations to describe the inter- and intra-phase strain fluctuations and can be evaluated by mean-field homogenization theories (for instance, see $[34,35]$ ) without any recourse to costly numerical simulations. Accordingly, most of the linearization procedures available for nonlinear elastic composites with isotropic constitutive phases - which is the case of all the linearization schemes considered in this work - linearize the constitutive behavior around reference strain(s) corresponding to one or more of these four descriptors of the strain field statistics. For such situations, the nonlinear relations $(5)_{1}$ and $(6)_{2}$ only depend on these four descriptors, i.e. $L^{r}, \tau^{r}, \omega_{a p p}^{r}$ are known nonlinear analytical functions of these four descriptors. For brevity, their definitions as well as the main concepts of the linearization procedures to which they are associated and on which all the enhanced linearization schemes presented in Section 3 are based, are recalled in Appendix B.

\subsection{Non-biased evaluation of linearization procedures}

As nonlinear homogenization schemes generate approximations for both the effective properties - overall responses or effective potentials - and moments of various orders - first, second or higher orders - of the local fields in nonlinear heterogeneous composites, it is important to evaluate their accuracy. However, such 
an evaluation can be either biased or non-biased. The main sources of bias are twofold: first, the use of different microstructures (e.g. [8]) to evaluate the effective behavior of both the exact nonlinear problem and the linear one related to the LCC derived from the linearization procedure. The second main type of bias is induced by the use of more or less accurate linear closed-form estimates - which are not exact results - to evaluate the effective properties of the LCC, e.g. [35, 41, 28, 16]. In order to get rid of such bias in the comparisons, objective methodology of comparison have been introduced recently and independently by Suquet and Moulinec [29] and Rekik et al. [37, 39]. The principle of this methodology is to evaluate the linearization procedures alone. For that, it relies on the analysis of an heterogenous unit cell or a volume element - e.g. with simple [37, 39] or random "periodic" [29, 21] microstructure - for which both the nonlinear homogenization problem and the linear homogenization problem associated with the chosen linear comparison composite (LCC) can be solved for exactly the same microstructure, the same loading conditions and with a similar and high level of accuracy by using the same numerical method, so that the effects of the sole linearization scheme can be evaluated without ambiguity.

In this paper, to design and evaluate new and enhanced nonlinear homogenization schemes, use is made of the objective comparison methodology presented by Rekik et al. and of its results $[39,40]$. In Rekik et al. works, the unit cell considered is a cylinder with a circular basis composed of a single spherical inclusion located at the cylinder's center and surrounded by matrix. As mentioned in Section 2, the nonlinear constituents are isotropic and their behaviors are assumed to be governed by a single potential (Eq. 1). Matrix and inclusion are perfectly bonded. In the ensuing calculations, the unit cell is subjected to a monotonic axisymmetric macroscopic deformation along the third direction - i.e. the cylinder axis - such that the overall strain is $\bar{\varepsilon}=\bar{\varepsilon}_{m} i+\iota \bar{\varepsilon}_{e q} \hat{e}$ where $\hat{e}=\hat{e}(\bar{\varepsilon})$ is reduced here to the axisymmetric tensor $e_{3} \otimes e_{3}-\frac{1}{2}\left(e_{1} \otimes e_{1}+e_{2} \otimes e_{2}\right)$ and $\iota$ is equal to +1 or -1 . A detailed expression of the $\mathrm{BC}$ are provided in [39]. Since the whole unit cell problem is fully invariant with respect to any rotation along the direction of the cylinder, the nonlinear local problem (3) associated with the heterogenous cylindrical unit cell is a 2D axisymmetric problem. Accordingly, the macroscopic stress $\bar{\sigma}$ is also axisymmetric and reads $\bar{\sigma}=\bar{\sigma}_{m} i+\frac{2}{3} \iota \bar{\sigma}_{e q} \hat{e}$ where $\bar{\sigma}_{m}$ and $\bar{\sigma}_{e q}$ are the overall hydrostatic and von Mises equivalent stresses.

At this stage, it should be emphasized that the 2D axisymmetric problem to be solved is a structural problem and not a local problem associated with a real material. Indeed, there is no composite material made of a periodic repetition of a cylindrical unit cell with a circular basis. Of course, the considered 2D axisymmetric problem can be viewed as an approximation of the classical 3D local problem associated with a real two-phase periodic composite submitted to an axisymmetric macroscopic strain and made of aligned spherical inclusions embedded in a matrix, these latter being distributed according to an hexagonal network in the transverse plane and aligned along the third direction. For such a two-phase periodic composite, a cylinder with an hexagonal basis with a single spherical inclusion located at the cylinder's center can be used as unit cell. In the following, all the linearization procedures will be tested on the $2 \mathrm{D}$ axisymmetric structural problem defined on the cylindrical unit cell with a circular basis. Namely, we compute the exact "up to numerical errors" solutions of both the nonlinear structural problem and the linear structural problems obtained when linearizing the constitutive behavior of the phase by means of the different meanfield homogenization linearization procedures. Therefore, even though the linearization procedures are not evaluated on a real composite but on a heterogenous cylinder with a circular basis, there is absolutely no bias in their evaluations since the results derived from the nonlinear homogenization schemes and the reference solution related to the $2 \mathrm{D}$ nonlinear structural problem are carried out on the same structure - i.e. the unit cell constituted by the heterogeneous circular cylinder. Furthermore, since the exact effective behavior of the circular cylindrical unit cell yields an accurate estimate of the effective behavior of the real above-described two-phase periodic composite with hexagonal unit cells $[20,25]$ and exhibits local field fluctuations very similar to those of the real periodic composite, the comparison between the various classical and proposed enhanced linearization procedures performed with these structural calculations will be representative of the 
relative performances of theses linearization schemes applied on real composites. By abuse of language the solutions related to the axisymmetric $2 \mathrm{D}$ structural problem will be referred to in the sequel as the solutions of the periodic two-phase composite.

For numerical applications, the constitutive law of the matrix is assumed as in [39] to obey to a RambergOsgood equation defined by

$$
\varepsilon_{m}=\frac{\sigma_{m}}{3 k^{\mathrm{m}}} \quad \text { and } \quad \varepsilon_{e q}=\frac{\sigma_{e q}}{3 \mu_{e}^{\mathrm{m}}}+\varepsilon_{0}\left(\frac{\sigma_{e q}}{\sigma_{0}}\right)^{n},
$$

where $k^{\mathrm{m}}$ and $\mu_{e}^{\mathrm{m}}$ are respectively the bulk and shear modulus of the elastic part of the matrix constitutive law. Besides, $m=1 / n$ is the work-hardening parameter ( $n$ is the nonlinearity exponent) such that $0 \leq m \leq$ $1, \varepsilon_{0}$ is an auxiliary strain, $\sigma_{0}$ is the flow stress. The inclusions are assumed to be linear isotropic elastic with bulk and shear moduli $k^{\mathrm{p}}$ and $\mu^{\mathrm{p}}$. Note that such type of composite enables to study the case of a rigidly-reinforced (resp. porous) material by assigning to the bulk and shear moduli of the inclusion (resp. pore) numerical values close to infinity (resp. zero).

\section{Proposed enhanced linearization procedures}

Based on some relevant observations and conclusions obtained from the evaluations of the secant, affine and second order procedures defined in Appendix B, especially those carried out by the non-biased comparative methodology of Rekik et al., we propose in this section to construct a set of six enhanced formulations for these linearization procedures. The key idea relies on the change of the reference strains used by these linearization schemes in order to generate novel and softer LCCs. The proposed alternative reference strains $\bar{\varepsilon}^{r}$ and $\hat{\varepsilon}_{e q}^{r}$ (instead of $\bar{\varepsilon}^{r}$ ) account for both inter- and intra-phase strain fluctuations. Note that this set of propositions is not exhaustive and could be enlarged in future works. As a first numerical experimentation of "new" nonlinear homogenization schemes with low additional numerical expense, the proposed formulations in this paper are limited to the change of the tensor of moduli $L^{r}$ in the phase of the LCC. Other formulations, more enriched, can be obtained by proposing new polarization tensor $\tau^{r}$ as it is the case for the LS formulation.

In what follows, for each proposed enhanced formulation, we first recall the limitations and advantages of the existent linearization schemes which have led to their definition and then report the (thermo-)elastic moduli for each individual constituents of the enhanced LCCs. Next, we consider the advantages and possible limitations of the proposed linearization procedure. Their practical efficiency is tested later in Section 4.1.

\subsection{Modified secant formulation "SEC-K $K_{\text {cov }}$ "}

The secant formulations, especially the classical one (SEC), were shown $[12,11,41]$ to yield too stiff overall estimates. Even though the proposed modified extension VAR - proposed as an alternative to the SEC model - which takes the second moment of the strain field as the reference strain significantly improves the results of SEC since it provides a softer overall behaviour, it still remains stiffer than the reference solution consistently with the fact it yields an upper bound on the effective potential. In order to further soften the prediction of such secant formulations, one may be tempted to choose a reference strain that is even larger than the second moment. We propose here to replace the second moment $\bar{\varepsilon}_{e q}^{r}$ of the strain field in phase $r$ by the strain descriptor $\hat{\varepsilon}_{e q}^{r}$ which accounts for both the interphase and anisotropic intraphase strain fluctuations. It is easy to check that $\hat{\varepsilon}_{e q}^{r}$ is higher than $\bar{\varepsilon}_{e q}^{r}$ and $\bar{\varepsilon}_{e q}^{r}$ - the reference strains of the VAR

and SEC models, respectively - since $\left(\hat{\varepsilon}_{e q}^{r}\right)^{2}=\left(\overline{\bar{\varepsilon}}_{e q}^{r}\right)^{2}+2 \bar{\varepsilon}_{e q}^{r} \sqrt{\frac{2}{3} E:: C_{\varepsilon}^{r}}$. In addition, since the power-law "stress-strain" relationship is concave, the inequality $\hat{\varepsilon}_{e q}^{r}>\bar{\varepsilon}_{e q}^{r}>\bar{\varepsilon}_{e q}^{r}$ verified by the three strain descriptors 
implies the following one $\mu_{s c t}^{r}\left(\hat{\varepsilon}_{e q}^{r}\right)<\mu_{s c t}^{r}\left(\bar{\varepsilon}_{e q}^{r}\right)<\mu_{s c t}^{r}\left(\bar{\varepsilon}_{e q}^{r}\right)$ for the secant shear moduli. Accordingly, the modified secant formulation, referred hereafter to as "SEC- $K_{\operatorname{cov}}$ ", which makes use of an isotropic tensor of elastic moduli for each individual constituents of the LCC given by

$$
L^{r}=3 k^{r} J+2 \mu_{s c t}^{r}\left(\hat{\varepsilon}_{e q}^{r}\right) K
$$

and defines the overall effective properties by the averages of strains and stresses in the LCC, should lead to softer estimates than those provided by the VAR formulation.

As defined previously (see Eqs. (12) and (14)), the strain descriptor $\hat{\varepsilon}_{e q}^{r}$ requires the calculation of the parallel and perpendicular measures of the strain fluctuations in phase $r$ which are defined for an anisotropic phase $r$ in the LCC. Accordingly, even though the SEC-K $\mathrm{K}_{\text {cov }}$ proposition apparently defines isotropic elastic phases as shown in Eq. (16), it implicitly requires the definition of anisotropic phases since it makes use of the measures of the strain fluctuations in the phases, the calculation of which by means of mean-field homogenization theories requires an anisotropic model as shown for instance by formulae (8) and (9) in [18]. The SEC-K $\mathrm{K}_{\text {cov }}$ approach may thus allow to improve the VAR estimates, in the sense it generates softer estimates, at low additional analytical and numerical expenses only generated by the computation of the parallel and perpendicular measures of the strain fluctuations in the phases.

Another advantage of this model is that it should not yield linear overall estimates for porous materials under hydrostatic loading, as it is the case for the SEC, AFF-ANI and AFF-ISOT schemes [24, 5, 39]. At last, note that the overall estimate of the proposition SEC-K $\mathrm{K}_{\text {cov }}$ coincide with that of the VAR model in the case of isotropic porous material under pure hydrostatic loading. This can be easily deduced from the definitions of the strain descriptors $\bar{\varepsilon}_{e q}^{r}$ (see Eq. (13)) and $\hat{\varepsilon}_{e q}^{r}$ (Eq. (14)) as functions of the covariance tensor $C_{\varepsilon}^{r}(11)_{2}$ which does not vanish unlike the deviatoric tensor $\bar{\varepsilon}_{d}^{r}$ which is identically null in this case. This model does however not lead to a bound neither to an effective potential.

\subsection{First enhanced affine formulation "AFF-ANI-F "}

Evaluative methodologies relying on comparisons between estimates and available bounds (e.g. [24]) have shown that the classical affine gives too stiff estimates. This result was confirmed by the objective evaluations carried out in [39] by Rekik et al. where it is observed that the original AFF-ANI formulation highly overestimates the "exact" overall response for all ranges of the work-hardening exponent $\mathrm{m}$. In the same study, it is shown that its simplified version AFF-ISOT gives estimates in good agreement with the reference global response for non-vanishing values of $m$ but underestimates the reference global response for strong nonlinearities thus showing that the AFF-ISOT is on a whole too soft. On the other hand, it was concluded in the same study that a linearization procedure making use of an anisotropic thermoelastic LCC provides better results than those relying on an elastic LCC with isotropic moduli. All these results suggest to define an enhanced intermediate affine version, denoted in the following by "AFF-ANI- $F_{\alpha}$ ", lying between the AFF-ANI and AFF-ISOT formulations by means of a linear combination of both LCCs of the AFF-ANI and AFF-ISOT versions, i.e. $L_{A F F-A N I-F_{\alpha}}^{r}=\alpha L_{A F F-I S O T}^{r}+(1-\alpha) L_{A F F-A N I}^{r}$ with $\alpha \in[0,1]$. The anisotropic thermoelastic stiffness tensor in each individual constituents of the enhanced LCC reads then

$$
L^{r}=3 k^{r} J+2 \mu_{t g t}^{r}\left(\bar{\varepsilon}_{e q}^{r}\right) E^{r}+2\left(\alpha \mu_{t g t}^{r}\left(\bar{\varepsilon}_{e q}^{r}\right)+(1-\alpha) \mu_{s c t}^{r}\left(\bar{\varepsilon}_{e q}^{r}\right)\right) F^{r}
$$

where, in this study, $\alpha$ is empirically adjusted so as to minimize the difference between the estimates of AFF-ANI- $F_{\alpha}$ and the exact overall response for an extreme situation corresponding to strong nonlinearities $(0 \leq m \leq 0.2)$. The new polarization tensor $\tau^{r}$ is defined as for the AFF-ANI formulation by Eq. $(30)_{2}$ but by substituting the standard tangent operator $(30)_{1}$ by the enhanced one (17). Note that the choices $\alpha=0$ and $\alpha=1$ corresponds to the AFF-ANI and AFF-ISOT formulations, respectively. 
The principle of this formulation is similar to that of some variants of the tangent and classical secant schemes $[27,26,1]$ based on the introduction of a multiplicative coefficient or a tuning parameter in the phases/matrix interaction law allowing to improve the self-consistent results for viscoplastic polycristalline metals. Moreover, this tuning parameter is fitted to finite element results available in the literature. Eventually, even though this formulation is empirical, it does not lead to a bound, neither defines an effective potential, nor incorporates the intra-phase fluctuations of the local strain field. Furthermore, it leads to linear overall estimates for isotropic porous materials under hydrostatic loading. However, it presents the advantage to be numerically easy to compute.

\subsection{Second enhanced affine formulation "AFF-ANI-F cov"}

As mentioned in Section 3.2, it was shown in [39] that a "stress-strain" linearization procedure which makes use of an anisotropic thermoelastic LCC moduli yields better estimates than those relying on an elastic LCC with isotropic moduli (if compared approaches include the same degree of information regarding the statistics of the local fields).

Moreover, for quasi-rigidly reinforced power-law composites with strongly nonlinear matrix, the analysis of the parallel measures of the stress fluctuations over the matrix $[38,40]$ have shown that they are significantly overestimated by the secant formulations (SEC and VAR) ((i)-(a)) and strongly underestimated by the affine version of the second-order procedure SOE-2 especially for $m<0.15$ ((i)-(b)) (for instance, see Figure 5-a in A.2). However it was demonstrated that the LS and AFF-ISOT schemes provide good estimates for these fluctuations in the nonlinear composite ((i)-(c)), even though they slightly under-estimate them for strong nonlinearities. According to Eq. $(27)_{1}$ and to the fact that the predictions of the measures of the strain fluctuations in the parallel direction over the matrix are quantitatively very similar for almost all linearization schemes (see Figure 6-a in A.2 and Figure 11-(ii.a) in [39]), it is possible to deduce from (i)-(a) that the secant shear moduli $\mu_{s c t}^{r}\left(\bar{\varepsilon}_{e q}^{r}\right)$ and $\mu_{s c t}^{r}\left(\bar{\varepsilon}_{e q}^{r}\right)$ are too stiff estimates for the shear modulus of a relevant enhanced LCC in the parallel direction of the tangent operator in the individual phases. In contrast, the shear modulus along the projector $E^{r}$ in the generalized secant tensor (defined by equation (32)) is a very soft estimate (see (i)-(b)) for this shear modulus. At last, it appears that the tangent shear modulus $\mu_{t g t}^{r}\left(\bar{\varepsilon}_{\text {eq }}^{r}\right)$ is a quite good estimate (see (i)-(c)) for the shear modulus along the projector $E^{r}$ in the tangent operator of the phase $r$ in the LCC.

On the other hand, the analysis of the orthogonal measures of the stress fluctuations over the matrix (see Figure 5-b in A.2) have shown that the AFF-ISOT scheme excessively underestimates them ((ii)-(a)) unlike the AFF-ANI and LS schemes which strongly overestimate them ((ii)-(b)). Moreover, the affine version of the second-order (SOE-2) and VAR procedure provide the closest estimates to these fluctuations in the matrix of the nonlinear composite ((ii)-(c)). According to Eq. (27) $)_{2}$ and to the fact that the orthogonal measures of the strain fluctuations over the matrix are very close for all the linearization schemes considered in this study (see Figure 6-b in A.2 and Figure 11-iii.a in [39]), one can concluded that the tangent shear modulus $\mu_{t g t}^{r}\left(\bar{\varepsilon}_{e q}^{r}\right)$ is a very soft estimate (see (ii)-(a)) for the shear modulus of a relevant enhanced LCC in the orthogonal direction of the tangent operator of the phase $r$. This later also appears to be highly overestimated by the secant shear modulus $\mu_{s c t}^{r}\left(\bar{\varepsilon}_{e q}^{r}\right)$ (see (ii)-(b)). Finally, the moduli $\mu_{s c t}^{r}\left(\bar{\varepsilon}_{e q}^{r}\right)$ and $\mu_{s c t}^{r}\left(\hat{\varepsilon}_{e q}^{r}\right)$, which incorporate both the inter- and intraphase strain fluctuations and respectively correspond to the secant moduli along $F^{r}$ in the LCC generated by the VAR and SOE-2 procedures, seem to be appropriate estimates (see (ii)-(c)) for the shear modulus along the projector $F^{r}$ in the phase $r$ of the LCC.

The combination of these conclusions derived at the global and local scales from the study of the reinforced case, motivate the proposition of new affine formulations (two variants) which define an anisotropic LCC 
with the following stiffness tensors

$$
L^{r}=3 k^{r} J+2 \mu_{t g t}^{r}\left(\bar{\varepsilon}_{e q}^{r}\right) E^{r}+2 \mu_{s c t}^{r}\left(\hat{\varepsilon}_{e q}^{r}\right) F^{r},
$$

or

$$
L^{r}=3 k^{r} J+2 \mu_{t g t}^{r}\left(\bar{\varepsilon}_{e q}^{r}\right) E^{r}+2 \mu_{s c t}^{r}\left(\bar{\varepsilon}_{e q}^{r}\right) F^{r} .
$$

Again, the polarization tensor $\tau^{r}$ is defined as for the AFF-ANI formulation by Eq. (30) 2 but by substituting the standard tangent operator (30) 1 by the enhanced one (18) or (19).

Since the first proposition (18) is expected to overestimate the exact nonlinear overall response and that the second one (19) should yield estimate stiffer than that of the proposition (18) - recall that $\mu_{s c t}^{r}\left(\hat{\varepsilon}_{\text {eq }}^{r}\right)<$ $\mu_{s c t}^{r}\left(\overline{\bar{\varepsilon}}_{e q}^{r}\right)$, our attention will be only focused on the first formulation (18) which will be investigated in this work. In the sequel, the formulation corresponding to the tangent operator (18) will be denoted by "AFFANI- $F_{\text {cov }}$ ". Note that this proposition explicitly incorporates information on both the inter- and intra-phase anisotropic strain fluctuations in the phases. This could soften the evaluations of the classical affine which only incorporates the interphase strain fluctuations through their first-order moments. Moreover, when compared to the original AFF-ANI scheme, the proposition (18) requires low additional numerical expenses. It should be noted that the "AFF-ANI- $F_{\text {cov }}$ " formulation is close but not similar to the affine version of the SOE-2 approach. Indeed, following Eq. $(17)_{2}$ in [15], it is noted for the case of isotropic, incompressible phases that the shear modulus in the orthogonal direction (along $F^{r}$ ) in the tangent operator (18) is identical to that defined by the SOE-2 scheme in the same direction. However, this is no longer the case for the shear modulus in the parallel direction (along $E^{r}$ ) since its definition (see Eq. $(17)_{1}$ in [15]) is more complex for the SOE-2 formulation - and also more relevant since it includes the intraphase fluctuations (see paragraph below) - than for the "AFF-ANI- $F_{\text {cov }}$ " approach (see Eq. (18)).

On the other hand, since the deviatoric part of the phase averages of the strain field vanish for isotropic porous materials under hydrostatic loading, it is relevant to consider alternatives for these enhanced formulations based on the replacement of the standard tangent shear modulus $\mu_{t g t}^{r}\left(\bar{\varepsilon}_{e q}^{r}\right)$ along the projector $E^{r}$ by the following ones $\mu_{t g t}^{r}\left(\bar{\varepsilon}_{e q}^{r}\right)$ or $\mu_{t g t}^{r}\left(\hat{\varepsilon}_{e q}^{r}\right)$ since the strain descriptors $\bar{\varepsilon}_{e q}^{r}$ and $\hat{\varepsilon}_{e q}^{r}$ do not vanish in this limiting case. For simplicity, we only mentioned the alternative tangent operators to the AFF-ANI-F $\operatorname{cov}$ formulation (Eq. (18)) which read:

$$
L^{r}=3 k^{r} J+2 \mu_{t g t}^{r}\left(\bar{\varepsilon}_{e q}^{r}\right) E^{r}+2 \mu_{s c t}^{r}\left(\hat{\varepsilon}_{e q}^{r}\right) F^{r}
$$

or

$$
L^{r}=3 k^{r} J+2 \mu_{t g t}^{r}\left(\hat{\varepsilon}_{e q}^{r}\right) E^{r}+2 \mu_{s c t}^{r}\left(\hat{\varepsilon}_{e q}^{r}\right) F^{r}
$$

These alternative propositions, which by definition are expected to soften the results derived from the AFF-ANI-F ${ }_{\text {cov }}$ formulation, are left for future investigation.

\subsection{Enhanced tangent second-order procedures $S O E-1-F_{c o v}, S O E-1-F_{m o m_{2}}$ and $S O E-1-(E-F)_{c o v}$}

As shown by the results obtained by Rekik et al. [39] non-biased methodology, the tangent second-order procedure SOE-1 over-estimates the overall response for reinforced composite and porous media. Moreover, unlike the SOE-2 procedure, the tangent second-order scheme fails to reproduce the evolution of the exact solution in the range of strong nonlinearities. It is recalled that such limitations were explained by the fact that SOE-1 does not explicitly account for the intraphase strain fluctuations as it makes use of the classical tangent stiffness tensor for the actual phase in the LCC taken at $\varepsilon^{r}=\bar{\varepsilon}^{r}$ (Eq. (33) 1 ). Accordingly, in order to soften the overall estimates of the tangent second-order SOE-1, we propose, as for the affine formulation, to soften the standard secant shear modulus $\mu_{s c t}^{r}\left(\bar{\varepsilon}_{e q}^{r}\right)$ of the tangent operator in the orthogonal direction for each individual phase of the LCC by substituting it with a softer shear modulus $\mu_{s c t}^{r}\left(\bar{\varepsilon}_{e q}^{r}\right)$ or $\mu_{s c t}^{r}\left(\hat{\varepsilon}_{e q}^{r}\right)$ 
incorporating both inter- and intra-phase strain fluctuations. Moreover, for the same reasons mentioned for the enhanced affine formulations, we choose in a first step to retain the standard shear tangent modulus $\mu_{t g t}^{r}\left(\bar{\varepsilon}_{e q}^{r}\right)$ for the parallel direction of the tangent operator. Thus, the thermoelastic LCCs associated with the enhanced SOE-1 approaches are defined by Eqs. (18) or (19) for their respective anisotropic stiffness tensor $L^{r}$, and by Eq. $(30)_{2}$ for their polarization tensor $\tau^{r}$. Once the choice of the LCC is performed, to complete the design of enhanced SOE-1 approaches we followed the same procedure as the one used by Ponte Castañeda in [33] for the SOE-1 model. Accordingly, the strain potential $w^{r}$ in each phase $r$ is approximated by a second-order Taylor expansion around a reference strain $\varepsilon^{r}$ set to the strain average over the phase $\bar{\varepsilon}^{r}$ within the LCC such that

$$
w^{r}(\varepsilon) \simeq w_{a p p}^{r}(\varepsilon)=w^{r}\left(\varepsilon^{r}\right)+\frac{\partial w^{r}}{\partial \varepsilon}\left(\varepsilon^{r}\right):\left(\varepsilon-\varepsilon^{r}\right)+\frac{1}{2}\left(\varepsilon-\varepsilon^{r}\right): L^{r}:\left(\varepsilon-\varepsilon^{r}\right)
$$

where $L^{r}$ is defined by (18) or (19). The potential $w_{a p p}^{r}(\varepsilon)$ can be rewritten as

$$
w_{a p p}^{r}(\varepsilon)=w^{r}\left(\varepsilon^{r}\right)+\frac{\partial w^{r}}{\partial \varepsilon}\left(\varepsilon^{r}\right): \varepsilon^{r}+\frac{1}{2} \varepsilon^{r}: L^{r}: \varepsilon^{r}+\left(\left(\frac{\partial w^{r}}{\partial \varepsilon}\left(\varepsilon^{r}\right)-L^{r}: \varepsilon^{r}\right): \varepsilon+\frac{1}{2} \varepsilon: L^{r}: \varepsilon\right) .
$$

As expected, the local constitutive law $\sigma=\frac{\partial w_{a p p}^{r}}{\partial \varepsilon}(\varepsilon)$ derived from the potential $w_{a p p}^{r}(\varepsilon)$ corresponds to the behavior of the enhanced SOE-1 thermoelastic LCC characterized by $\sigma=L^{r}: \varepsilon+\tau^{r}$ with $L^{r}$ and $\tau^{r}$ defined respectively by (18) or (19) and $(30)_{2}$, since $\varepsilon^{r}=\bar{\varepsilon}^{r}$. The main difference with the SOE-1 model is that the stiffness tensor $L^{r}$ of the derived LCC differs from the tangent stiffness tensor $L_{t g t}^{r}\left(\varepsilon^{r}\right)=\frac{\partial^{2} w^{r}}{\partial \varepsilon^{2}}\left(\varepsilon^{r}\right)$ and is equal to (18) or (19). Then, similarly to the SOE-1 model, the effective potential or stored-energy function $\tilde{w}(\bar{\varepsilon})$ of the nonlinear composite may be approximated as follows

$$
\tilde{w}(\bar{\varepsilon})=\min _{\varepsilon \in \kappa(\bar{\varepsilon})}<w(\varepsilon)>\simeq \tilde{w}_{a p p}(\bar{\varepsilon})=\min _{\varepsilon \in \kappa(\bar{\varepsilon})}\left(\sum_{r=1}^{N} f^{r}<w_{a p p}^{r}(\varepsilon)>^{r}\right)
$$

The optimal value of the effective potential $\tilde{w}(\bar{\varepsilon})$ is obtained when the stationarity conditions of this potential with respect to the parameters $\varepsilon^{r}$ are ensured. These stationarity conditions are satisfied for $\varepsilon^{r}=\bar{\varepsilon}^{r}$ which corresponds to the initial choice we made to define $w_{a p p}^{r}(\varepsilon)$ in Eq. (22). Indeed, as shown in [34], the stationarity of $\tilde{w}(\bar{\varepsilon})$ with respect to $\varepsilon^{r}$ reads

$$
\frac{\partial \tilde{w}}{\partial \varepsilon^{r}}(\bar{\varepsilon})=f^{r}\left(L^{r}-\frac{\partial^{2} w^{r}}{\partial \varepsilon \partial \varepsilon}\left(\varepsilon^{r}\right)\right)\left(\varepsilon^{r}-<\varepsilon>^{r}\right)=0,
$$

thus leading to the choice $\varepsilon^{r}=\bar{\varepsilon}^{r}$. The later reference strain allows to estimate the effective potential of the nonlinear composite by (31). As mentioned in [34], this estimation is available for any choice of the tensor of moduli $L^{r}$. For the SOE-1 procedure, in order to determine an optimal choice for the tensor $L^{r}$, Ponte Castañeda tried to ensure the stationarity of $\tilde{w}(\bar{\varepsilon})$ with respect to $L^{r}$. As the later condition have not led to useful results $[33,5]$, he made the choice of the tangent stiffness tensor (30). Other possible alternatives for this tensor are the propositions (18) or (19) stemming from the analysis of the results obtained by Rekik et al. in their evaluative studies [39, 40]. At this stage, it should be emphasized that the reference strain $\overline{\bar{\varepsilon}}^{r}$ associated with proposition (19) is evaluated for an anisotropic thermoelastic LCC - unlike the case of the VAR method for which the LCC is elastic and isotropic - following Eq. $(13)_{2}$. At last, the method to evaluate the effective response is similar to that followed for the SOE1 method, i.e. based on the construction of an effective potential estimated by $\tilde{w}(\bar{\varepsilon}) \simeq \sum_{r} f^{r} w_{a p p}^{r}\left(\bar{\varepsilon}^{r}\right)=$ $\min _{\varepsilon \in \kappa(\bar{\varepsilon})}\left(\sum_{r} f^{r}\left(w^{r}\left(\varepsilon^{r}\right)+\frac{1}{2} L^{r}::\left\langle\left(\varepsilon-\varepsilon^{r}\right) \otimes\left(\varepsilon-\varepsilon^{r}\right)\right\rangle^{r}\right)\right)=\sum_{r} f^{r}\left(w^{r}\left(\bar{\varepsilon}^{r}\right)+\frac{1}{2} \frac{\partial w^{r}}{\partial \varepsilon}\left(\bar{\varepsilon}^{r}\right):\left(\bar{\varepsilon}-\bar{\varepsilon}^{r}\right)\right)$, from which the effective stress is obtained according to $\bar{\sigma}=\frac{\partial \tilde{w}}{\partial \bar{\varepsilon}}(\bar{\varepsilon})$. 
Even though the proposed modified tangent second-order schemes partially satisfy the stationarity conditions - only that with respect to $\varepsilon^{r}$ as it is the case for the SOE-1 model but not with respect to $L^{r}$, they are expected to soften the global response of the tangent second-order procedure and hence to improve on the results of this scheme since these propositions explicitly account for the intraphase strain fluctuations in contrast to the SOE-1 model. Hereafter, the energetic formulations associated with the affine schemes AFF-ANI-F cov $_{\text {and AFF-ANI-F }}$ mom $_{2}$, respectively associated with the stiffness tensors defined by (18) and (19), are referred to as SOE- $1-F_{\text {cov }}$ and SOE- $1-F_{m o m_{2}}$, respectively.

Other alternatives for these enhanced energetic formulations are the second-order extensions of the enhanced affine formulations (20) and $(21)^{1}$ where the standard tangent shear moduli $\mu_{t g t}^{r}\left(\bar{\varepsilon}_{e q}^{r}\right)$ along the projector $E^{r}$ have been replaced by the following ones $\mu_{t g t}^{r}\left(\bar{\varepsilon}_{e q}^{r}\right)$ and $\mu_{t g t}^{r}\left(\hat{\varepsilon}_{e q}^{r}\right)$ since the first-order moments of the strain over the phase $\bar{\varepsilon}_{e q}^{r}$ vanish for isotropic porous materials under purely hydrostatic loading unlike the strain descriptors $\bar{\varepsilon}_{e q}^{r}$ and $\hat{\varepsilon}_{e q}^{r}$. These alternative formulations are expected to provide better overall estimates namely a pronounced nonlinear behavior - for porous materials under hydrostatic loading than the SEC, AFF-ANI, AFF-ISOT and enhanced affine formulations (Eqs. (18) and (19)). For the sake of simplicity, only the enhanced energetic formulation associated with the affine formulation (21) and referred to as SOE$1-(\mathrm{E}-\mathrm{F})_{\text {cov }}$ will be considered in the sequel.

\section{A first evaluation of the performance of the proposed enhanced formulations}

\subsection{Considered materials and numerical implementations}

Numerical experiments of the proposed enhanced LCCs are carried out for two different types of heterogeneous materials:

(i) "reinforced composites" composed of a nonlinear elastic matrix reinforced by linear isotropic quasi-rigid elastic particles,

(ii) "porous media" with a nonlinear elastic matrix.

All the considered nonlinear elastic individual constituents are assumed to obey Ramberg-Osgood constitutive equations as defined in Eq. (15).

For all the FE computations (except for very low pore volume fractions $f^{\mathrm{p}} \leq 1 \%$ where the mesh should be refined near the pores), the number of finite elements inside the matrix $N^{\mathrm{m}}$ and inside the inclusion $N^{\mathrm{p}}$ has been set to $N^{\mathrm{m}}=900$ and $N^{\mathrm{p}}=300$, respectively. Such a mesh has been shown in [39] to be sufficiently refined to guarantee a good convergence of the numerical solution both in terms of the effective properties and local fields fluctuations. In the hereafter reported illustrative results, the material parameters have been chosen as indicated in Table 1. Additional parameters such as $f^{\mathrm{p}}$ and $m$ are specified when necessary.

\begin{tabular}{|l|c|c|c|c|c|c|}
\hline Material parameters & $E^{\mathrm{m}}$ & $\nu^{\mathrm{m}}$ & $\sigma_{0}^{\mathrm{m}}$ & $\varepsilon_{0}$ & $E^{\mathrm{P}}$ & $\nu^{\mathrm{p}}$ \\
\hline Reinforced composites & $75 \mathrm{GPa}$ & 0.3 & $300 \mathrm{MPa}$ & $100 \%$ & $400 \mathrm{GPa}$ & 0.2 \\
\hline Porous media & $75 \mathrm{GPa}$ & 0.3 & $300 \mathrm{MPa}$ & $100 \%$ & - & - \\
\hline
\end{tabular}

Table 1: Parameters used for the finite element calculations

For the porous case the effective response is compressible, even if the matrix is almost incompressible, and is characterized by a two-dimensional relation $\left(\bar{\sigma}_{m}, \bar{\sigma}_{e q}\right)=\tilde{f}\left(\bar{\varepsilon}_{m}, \bar{\varepsilon}_{e q}\right)$ since, as mentioned in Section 2.4, the

\footnotetext{
${ }^{1}$ These extensions are generated in a way similar to the SOE-1- $F_{\text {cov }}$ and SOE-1- $F_{m o m_{2}}$ second-order procedures
} 
macroscopic strain and stress are axisymmetric and read $\bar{\varepsilon}=\bar{\varepsilon}_{m} i+\iota \bar{\varepsilon}_{e q} \hat{e}, \bar{\sigma}=\bar{\sigma}_{m} i+\frac{2}{3} \iota \bar{\sigma}_{e q} \hat{e}$, respectively. Since the local constitutive relations in their asymptotic behavior - i.e. for large strain - are positively homogeneous function of the same degree $m$ (see Eq. $(15)_{2}$ ), the above-mentioned two-dimensional relation can be rewritten as $\bar{\sigma}_{m}=\hat{\sigma}_{0}\left(\tau_{\varepsilon}\right)\left(\frac{\bar{\varepsilon}_{m}}{\varepsilon_{0}}\right)^{m}$ or $\bar{\sigma}_{e q}=\tilde{\sigma}_{0}\left(\tau_{\varepsilon}\right)\left(\frac{\bar{\varepsilon}_{e q}}{\varepsilon_{0}}\right)^{m}$ and therefore restrict to one function $\hat{\sigma}_{0}\left(\tau_{\varepsilon}\right)$ or $\tilde{\sigma}_{0}\left(\tau_{\varepsilon}\right)$, where the ratio $\tau_{\varepsilon}=\bar{\varepsilon}_{m} / \bar{\varepsilon}_{e q}$ is the macroscopic strain triaxiality ratio. Indeed, if the local constitutive relations were exactly positively homogeneous functions of the same degree $m$, the local strain solution of this problem would be a positively homogeneous function of degree one of the imposed strain $\bar{\varepsilon}$, the local stress a positively homogeneous function of degree $m$ and so would be the overall stress [34]. In practice, $\tilde{\sigma}_{0}\left(\tau_{\varepsilon}\right)$ is determined on the linear part of the evolution of $\ln \left(\bar{\sigma}_{e q}\right)$ with respect to $\ln \left(\bar{\varepsilon}_{e q}\right)$ for large enough $\bar{\varepsilon}_{e q}$ (for instance at $\bar{\varepsilon}_{e q}=0.04$ under isochoric extension). The same methodology is applied to determine $\hat{\sigma}_{0}\left(\tau_{\varepsilon}\right)$.

On the other hand, for a reinforced composite, the macroscopic behavior may be considered as almost incompressible, since the bulk moduli $k^{r}$ will be chosen sufficiently large. As a consequence, the sole overall quantity of interest is the deviatoric response $\bar{\sigma}_{e q}=\tilde{f}\left(\bar{\varepsilon}_{e q}\right)=\tilde{\sigma}_{0}\left(\frac{\bar{\varepsilon}_{e q}}{\varepsilon_{0}}\right)^{m}$ which is characterized by a single scalar: the effective flow stress $\tilde{\sigma}_{0}$.

For the computation of the effective behavior associated with either the exact solution or the different tested linearization procedures as well as the statistical descriptors of the local strain fields $\bar{\varepsilon}^{r}, \overline{\bar{\varepsilon}}_{e q}^{r}, \hat{\varepsilon}_{\|}^{r}, \hat{\varepsilon}_{\perp}^{r}$ inside both the actual nonlinear composite and the various LCCs derived from the linearization procedures, the reader is advised to refer to $[39,40]$ where detailed and thorough explanations of these calculations are provided. We simply recall that the solutions of both the exact nonlinear problem (3) and its approximations (5) derived from the different linearization procedures are obtained by finite elements computations carried out through the FE code CAST3M [7]. Furthermore, the statistical descriptors of the strain fields within the LCC, which are used to determine the effective behavior, are not obtained from the derivation of the effective thermoelastic energy of the LCC with respect to the local moduli or polarizations as it is usually the case in mean-fields theories but are computed by spatially integrating the local fields in the phases, which is numerically more efficient than the derivation of the effective energy since the local fields are explicitly provided by the FE method.

\subsection{Results and discussions}

\subsubsection{Rigidly-reinforced composites}

The evolutions with respect to the work-hardening exponent $m$ of the normalized effective flow stresses derived from the proposed SEC- $K_{c o v}$, SOE-1- $F_{c o v}$, SOE-1- $F_{m o m} 2$ and SOE-1-(E-F) cov formulations are reported in Fig. 1-a together with the ones associated with the secant, affine and second-order procedures and with the nonlinear (NL) exact solutions. Similarly, Fig. 1-b depicts the evolutions with respect to the work-hardening exponent $m$ of the absolute differences $\frac{\tilde{\sigma}_{0}-\tilde{\sigma}_{0}^{N L}}{\tilde{\sigma}_{0}^{N L}}$ between the normalized effective flow stresses derived from the six enhanced proposed linearization schemes, the ones described in Sections B.1 and B.2 and the exact nonlinear solution. It is observed that the predictions of the AFF-ANI- $F_{c o v}$ are, as expected, much softer than those provided by the classical affine AFF-ANI formulation and the secant formulations (SEC, VAR), especially for low values of the work-hardening parameter m. For strong nonlinearities, this enhanced model yields estimates which are very close to those provided by the LS and SOE-1 schemes. However it remains less relevant than these potential-based formulations for moderate to low work-hardening exponents since it over-estimates their predictions. Accordingly, it could be an alternative model to the LS and SOE-1 schemes in the range of strong nonlinearity since it yields similar overall responses than these models and presents the advantage to be less difficult to implement. 
As observed in Fig. 1, the results derived from the SOE-1- $F_{m o m}$ and SOE-1- $F_{c o v}$ procedures again confirm

(a)

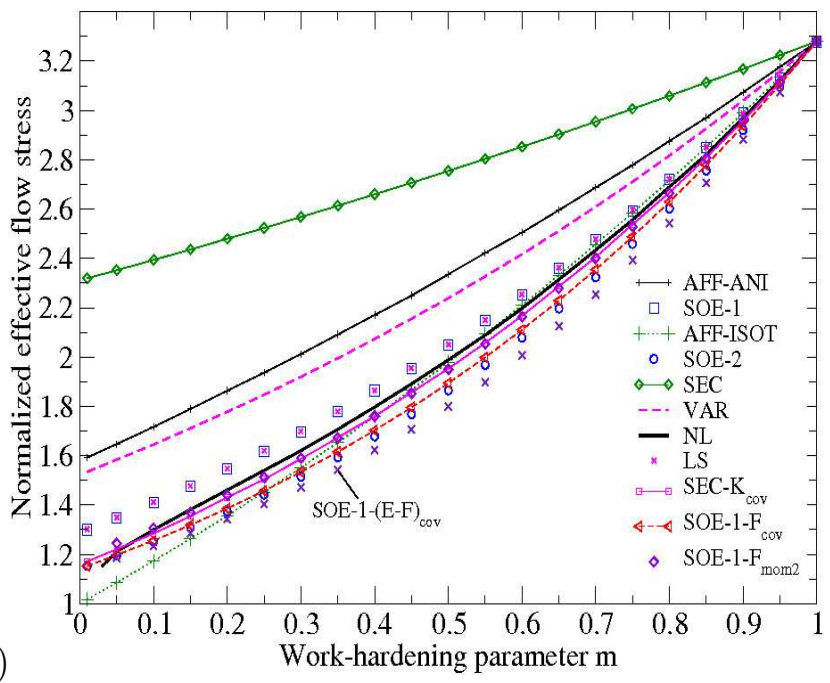

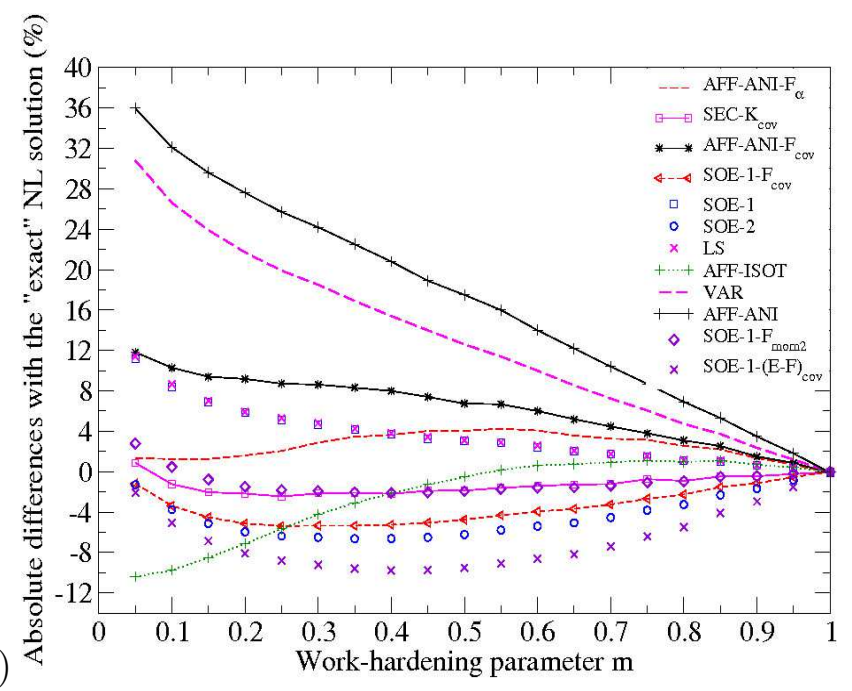

Figure 1: Reinforced composite: variation of (a) the normalized effective flow stress $\frac{\tilde{\sigma}_{0}}{\sigma_{0}^{\mathrm{m}}}$ associated with the linearization procedures $S E C-K_{\text {cov }}, S O E-1-F_{m o m 2}, S O E-1-F_{\text {cov }}$ and SOE-1-(E-F) cov and (b) the absolute differences $\frac{\tilde{\sigma}_{0}-\tilde{\sigma}_{0}^{N L}}{\tilde{\sigma}_{0}^{N L}}$ between the normalized effective flow stresses associated with the six enhanced linearization schemes and the exact solution with respect to the work-hardening exponent $m$. Results derived from the linearization schemes defined in Sections B.1 and B.2 are also reported for comparison purposes. $f^{\mathrm{p}}=0.3$ and $\alpha=3 / 4$.

the ability of the energetic formulations which linearize the local nonlinear behaviour at reference strains accounting for the intra-phase strain fluctuations to provide satisfactory estimates. Regarding the SOE-1$F_{\text {cov }}$ procedure, even though the overall responses provided by this formulation slightly over-estimate the predictions of the SOE-2 approach for moderate and low nonlinearities, they remain softer than the "exact" ones. This result was expected for the two following reasons. First, as implied by their respective definition, the tangent shear modulus $\mu_{t g t}^{r}\left(\bar{\varepsilon}_{e q}^{r}\right)$ used in the SOE-1- $F_{\text {cov }}$ formulation is slightly stiffer than the shear modulus adopted by the SOE-2 procedure in the parallel direction $E^{r}$ of the stiffness tensor (see Eq. $(17)_{1}$ in [15]). Second, the shear moduli in the orthogonal direction $F^{r}$ of the stiffness tensors respectively associated with the SOE-1- $F_{\text {cov }}$ and SOE-2 procedures are identical.

Concerning the SOE-1- $F_{m o m}$ enhanced formulation, as reported in Fig. 1-b, this approach provides accurate estimates for the overall exact solution even though it slightly over-estimates this latter when $m$ vanishes (the absolute error is less than $4 \%$ ). The SOE- $1-F_{m o m} 2$ procedure improves on the results of LS and SOE-1 for all values of the work-hardening exponents m. Moreover, it yields stiffer results than the SOE-2 formulation which slightly under-estimates the exact overall solution. We deduce from these observations and from the fact that the tangent shear modulus $\mu_{t g t}^{r}\left(\bar{\varepsilon}_{e q}^{r}\right)$ is a quite good estimate for the shear modulus along the projector $E^{r}$ (see section 3) and that the shear modulus $\mu_{s c t}^{r}\left(\overline{\bar{\varepsilon}}_{e q}^{r}\right)$ in the orthogonal direction of the tangent operator is a satisfactory estimate for low to moderate nonlinearity, i.e. $m \in[0.1,1]$, and should be slightly softer for high nonlinearity, i.e. when the work-hardening exponent $m$ vanishes, such as the shear modulus $\mu_{\text {sct }}^{r}\left(\hat{\varepsilon}_{e q}^{r}\right)$ used by the SEC-K $\mathrm{K}_{\text {cov }}$ and SOE-1-F $\mathrm{F}_{\text {cov }}$ models.

It is observed that the SOE-1-(E-F) cov model provides a (too) soft estimate compared to the NL solution. As expected, this model is also softer than the SOE-1- $F_{\text {cov }}$ since, by construction, the tangent shear modulus $\mu_{t g t}^{r}\left(\bar{\varepsilon}_{e q}^{r}\right)$ along the parallel direction $E^{r}$ of the SOE-1- $F_{\text {cov }}$ model is replaced by a softer tangent modulus $\mu_{t g t}^{r}\left(\hat{\varepsilon}_{e q}^{r}\right)$ inside the SOE-1-(E-F) cov approach. 
Fig. 1-b shows that the thermo-elastic anisotropic LCC used by the parametric AFF-ANI-F ${ }_{\alpha}$ formulation - with an empirically optimized value of the scalar parameter $\alpha$ set here to $\alpha_{0}=3 / 4$ - yields good estimates for strongly nonlinear power-law composites. It is observed (Fig. 1-b together with Fig. 1-a) that this formulation significantly improves on the predictions of the "stress-strain" SEC, VAR and AFF-ANI procedures. It also corrects the excessive under-estimation predicted by the AFF-ISOT approach for vanishing values of the work-hardening exponent $\mathrm{m}$. Accordingly, the shear modulus " $\alpha \mu_{t g t}^{r}\left(\bar{\varepsilon}_{e q}^{r}\right)+(1-\alpha) \mu_{\text {sct }}^{r}\left(\bar{\varepsilon}_{\text {eq }}^{r}\right)$ " associated with the orthogonal direction of the tangent operator for the optimized value $\alpha_{0}$ seems to be more appropriate than the shear moduli $\mu_{s c t}^{r}\left(\bar{\varepsilon}_{e q}^{r}\right)$ and $\mu_{t g t}^{r}\left(\bar{\varepsilon}_{e q}^{r}\right)$. This model could however be criticized because it does not account for the intra-phase strain fluctuations and would probably provide poor results for other situations where significant intra-phase strain fluctuations are observed as it is the case for large inclusion volume fractions [23]. Moreover, it slightly over-estimates the LS and SOE-1 predictions for low to moderate work-hardening exponents. Finally, the main advantage of this model is its simplicity and facility to be implemented as it does not require the computation of intra-phase field fluctuations.

As expected, it is observed in Fig. 1(a) that the modified secant formulation SEC- $K_{\text {cov }}$ provides softer estimates than the VAR approach. Moreover, this new formulation seems to capture the exact overall response of a rigidly-reinforced composite rather well, especially for vanishing work-hardening exponents $\mathrm{m}$, and yields better predictions, at least for these cases, than the AFF-ISOT formulation and "potential-based" approaches such as LS, SOE-1 and SOE-2. This result suggests that taking for reference strain associated with the secant shear modulus in the orthogonal direction $\left(F^{r}\right)$ the strain descriptor $\hat{\varepsilon}_{e q}^{r}$ - which accounts for both the first-moment of the strain field and the measure of the intra-phase strain fluctuations over the phase - might enable to define a relevant modified secant LCC.
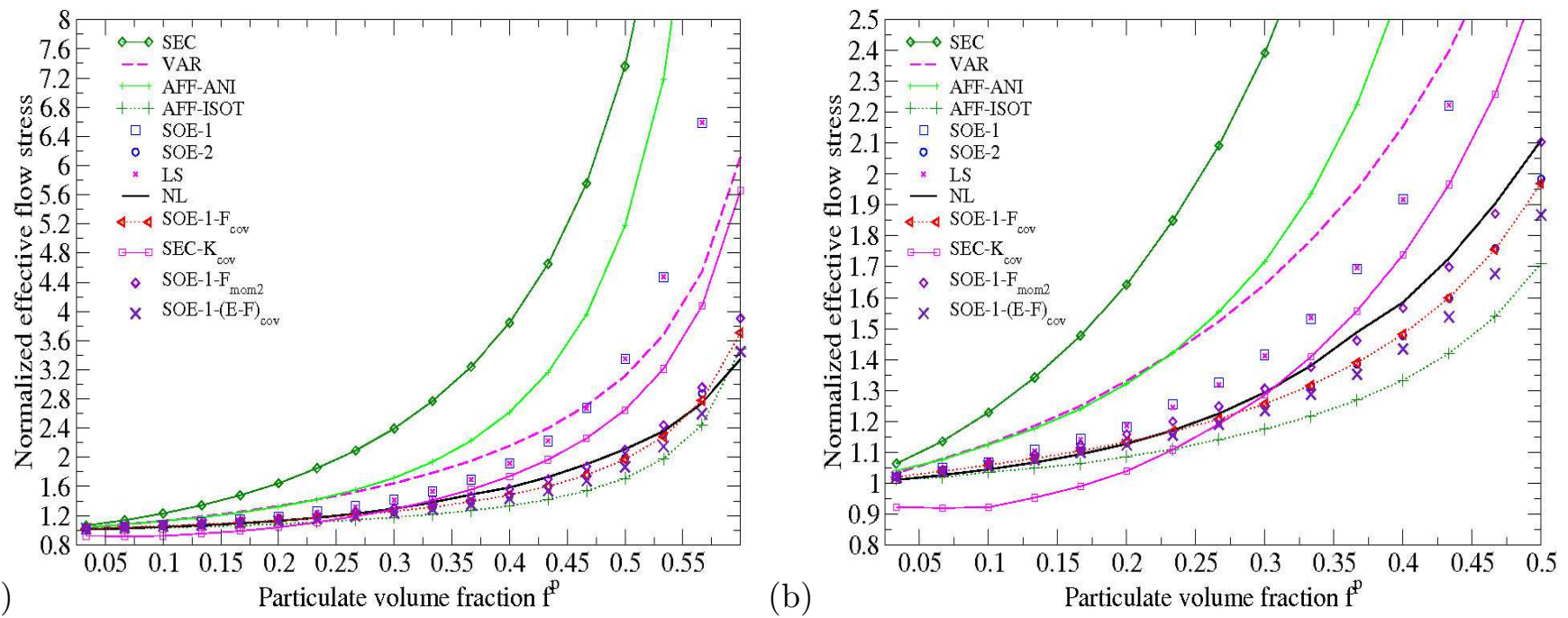

Figure 2: Reinforced composite: variation of the normalized effective flow stress $\frac{\tilde{\sigma}_{0}}{\sigma_{0}^{m}}$ associated with the linearization procedures $S E C-K_{\text {cov }}, S O E-1-F_{m o m} 2, S O E-1-F_{c o v}$ and $S O E-1-(E-F)_{\text {cov }}$ with respect to the particle volume fraction $f^{\mathrm{p}}$. Results derived from the linearization schemes defined in Sections B.1 and B.2 are also reported for comparison purposes. Full curves (a) and zoom (b). $m=0.1$.

Fig.(2) illustrates the evolutions of the normalized effective flow stress as functions of the particulate concentration $f^{\mathrm{p}}$ for strongly nonlinear reinforced power-law composites $(m=0.1)$. Note that among the six

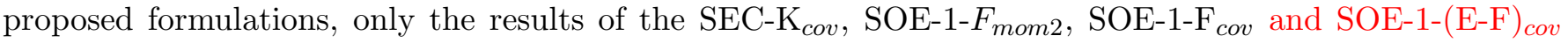
are reported since they have been seen to give the most satisfactory overall estimates for strongly nonlinear rigidly-reinforced composites. This figure confirms the trends observed in Fig. 1 when the particulate con- 
centration is less than 0.4. When $f^{\mathrm{p}}$ exceeds this limit and tends to the percolation threshold, it is observed that, even if the SEC- $K_{\text {cov }}$ approach improves on the results of the LS, SOE-1 and VAR formulations, it still yields stiffer results than the exact solution and SOE-2 schemes. Another surprising result with this secant formulation is observed for low volume fractions, below about $17 \%$, where it turns out that the effective properties of the reinforced composite as predicted by this model would be softer than those of the pure matrix: indeed the softening effect induced by the proposed new linearization is too strong with respect to the reinforcement effect due to the presence of rigid inclusions. This unphysical result together with the previous one illustrate the limitation of a simple secant formulation, which can hardly be accurate in all situations: the SEC- $\mathrm{K}_{\text {cov }}$ formulation is too soft for low volume fractions, and too stiff for higher ones, even though for intermediate ones, it captures well the dependance with nonlinearity. Any other specification for the reference strain associated with the secant modulus of the matrix, either lower or larger than $\hat{\varepsilon}_{e q}^{r}$, might also fail for some particular combination of parameters. This establishes new inherent limitations for the secant formulations.

In contrast with the SEC-K $\mathrm{K}_{\text {cov }}$ formulation, the predictions of the enhanced second-order formulations SOE-1- $F_{m o m}$, SOE-1- $F_{\text {cov }}$ and SOE-1-(E-F) $)_{\text {cov }}$ are in good agreement with the exact effective flow stress for all inclusion volume fractions even though the SOE-1-(E-F) cov estimate seems to underestimate NL for $f^{\mathrm{P}} \geq 0.3$ similarly to SOE-2 and SOE-1- $F_{\text {cov }}$. This result confirms the ability of these formulations to account for the intraphase strain fluctuations relevantly, even near the percolation limit. The performance of such formulations are likely to be linked to the higher sophistication of the linearization procedure, based on more parameters than the secant one: two moduli and a polarization are required to define anisotropic thermoelastic behaviors, while only one is used for an isotropic linear secant one.

\subsubsection{Porous media}

The enhanced formulations presented in Section 3 are now applied on a porous medium submitted to an axisymmetric isochoric extension and their predictions are reported in Fig. 3, for a pore concentration $f^{\mathrm{p}}=0.3$. It is observed that the $\mathrm{SEC}-\mathrm{K}_{\text {cov }}$ approach fails to predict accurately the overall response of the porous material since it yields an excessively soft overall response and an almost linear dependance of the effective critical stress with respect to $(m-1)$. Such a behavior is also observed for the other secant formulations (SEC and VAR schemes), with different slopes. An analytical proof of this property is provided in Appendix (C) and emphasizes a general limitation of secant formulations in such a situation.

The AFF-ANI-F ${ }_{\text {cov }}$ estimate (see Fig. 3) is seen to improve on the results of the SEC and AFF-ANI procedures, probably because it accounts for the intraphase strain fluctuations unlike the SEC and AFFANI formulations. Nevertheless, the AFF-ANI-F cov estimate still yields stiffer results than those provided by the VAR approach for moderate and strong nonlinearities. This might be explained by the fact that the effect of the intraphase strain fluctuations are only taken into account in the perpendicular direction (along $F^{r}$ ) of the stiffness tensor in the LCC while they are equally included in both directions, along $F^{r}$ and $E^{r}$, for the VAR scheme.

On the other hand, the AFF-ANI- $F_{\alpha}$ formulation, which does not incorporate the intra-phase fluctuations of the strain field, improves on the results of the VAR approach for non-vanishing values of $m$ (i.e. for $m>0.2$ ) but, as expected, leads to poor results when $m$ goes to 0 , i.e. when the intraphase strain fluctuations become large. Note that this formulation has been applied with $\alpha=\frac{3}{4}$ since this value leads to accurate estimates for strongly nonlinear power-law reinforced composites as seen in the previous paragraph. Another value of $\alpha$ would of course have generated other results. But it would be of poor interest to have to adjust a parameter for each combination of constitutive relation.

The evaluation of the enhanced tangent second-order formulation SOE-1-F $\mathrm{F}_{\text {cov }}$ confirms again its ability to 
reproduce the trends of the exact solution qualitatively and quantitatively. Indeed, although this enhanced SOE-1 formulation slightly under-estimates the "exact" solution, it yields better estimates than those predicted by the "stress-strain" approaches and improves on the predictions of the LS and SOE-1 schemes for vanishing values of $m$. Furthermore, it is observed for this situation that it also slightly improves on the SOE-2 procedure over the whole range of $m$ values. The same trends are observed for the SOE-1-F $\mathrm{F}_{\text {mom } 2}$ which turns out to provide the best estimate of the overall exact behavior among all tested formulations. This modified potential-based formulation provides as expected stiffer results than the SOE-1-F cov approach since its secant shear modulus $\mu_{s c t}^{r}\left(\overline{\bar{\varepsilon}}_{\text {eq }}^{r}\right)$ along the direction $F^{r}$ is stiffer than the one $\mu_{s c t}^{r}\left(\hat{\varepsilon}_{e q}^{r}\right)$ used by the SOE-1-F $\mathrm{F}_{\text {cov }}$ procedure. These tendencies confirm the relevance of the enhanced potential-based formula-

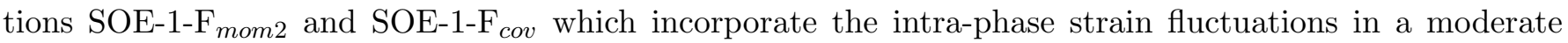
way (more than the SOE-1 or LS approaches but less than the SOE-2 procedure) to predict the overall behavior of both reinforced composites or porous materials. Note that the SOE-1-(E-F) cov estimate is not reported in Figure (3) since, by its definition, it is expected to be softer than the response of the SOE-1-F procedure which is already too soft.

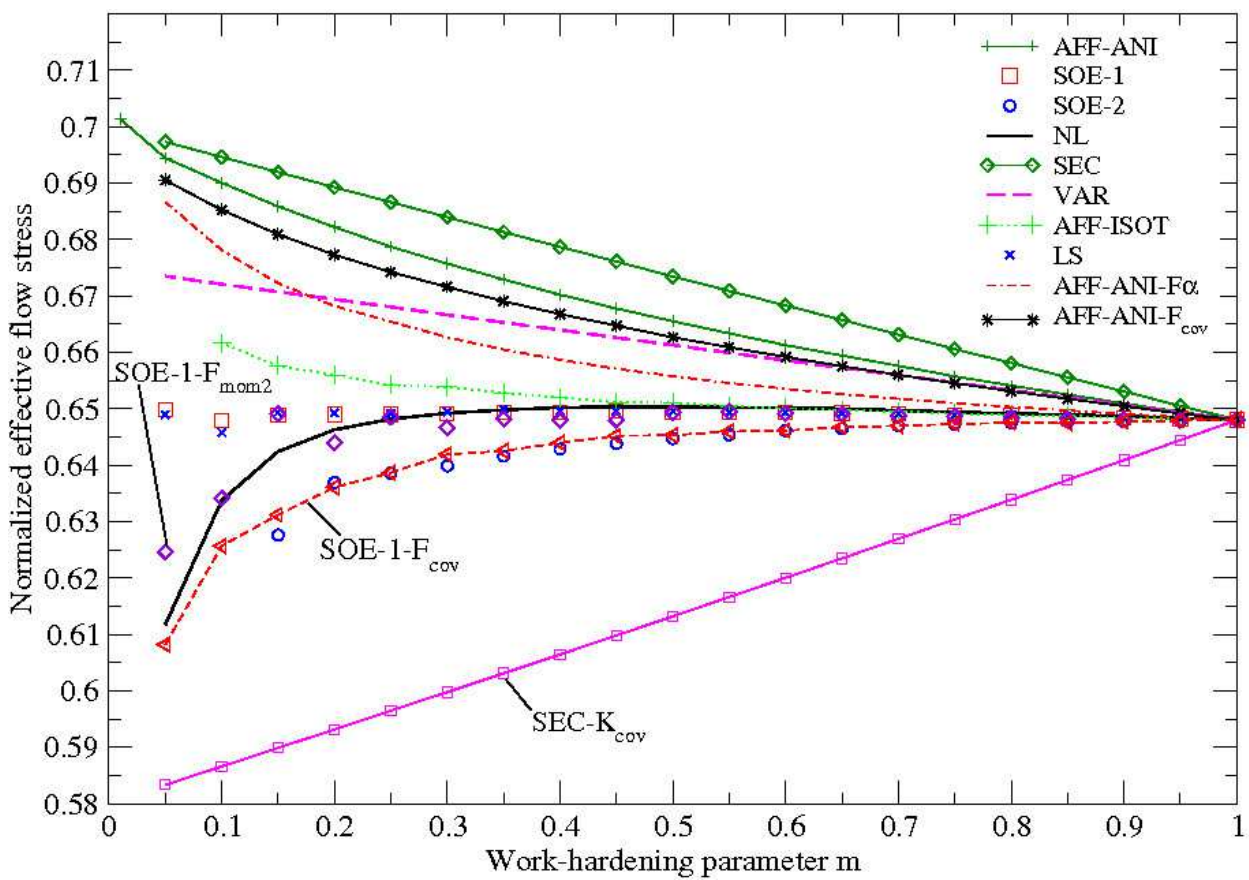

Figure 3: Porous material under isochoric extension: variation of the normalized effective flow stresses $\frac{\tilde{\sigma}_{0}}{\sigma_{0}^{\mathrm{m}}}$ associated with the five enhanced linearization procedures with respect to the work-hardening exponent $m$. Results derived from the linearization schemes defined in Sections B.1 and B.2 are also reported for comparison purposes. $f^{\mathrm{P}}=0.3$ and $\alpha=3 / 4$.

The predictions of the following five enhanced formulations (AFF-ANI-F ${ }_{\text {cov }}, \mathrm{SEC}_{\text {cov }}$, SOE-1-F cov, SOE$1-\mathrm{F}_{\text {mom } 2}$ and SOE-1-(E-F) cov $)$ are now tested on a very discriminant situation which consists of a porous medium submitted to a pure hydrostatic loading at a low pore concentration $f^{\mathrm{p}}=0.01$. Such a situation is classically representative of problems of ductile fracture $[22,31,43]$. The effective predictions of these linearization procedures are depicted in Figure (4). The results of the SEC, AFF-ANI, AFF-ISOT, SOE1 and LS models are not reported since they only make use of the first-order moment of the strain field $\bar{\varepsilon}_{e q}^{r}$ when defining the stiffness tensor $L_{r}$ in phase $\mathrm{r}$ in the LCC. For such an hydrostatic loading, $\bar{\varepsilon}_{e q}^{r}$ is close to zero inside the matrix for the considered unit cell. Accordingly, these models lead to effective flow 

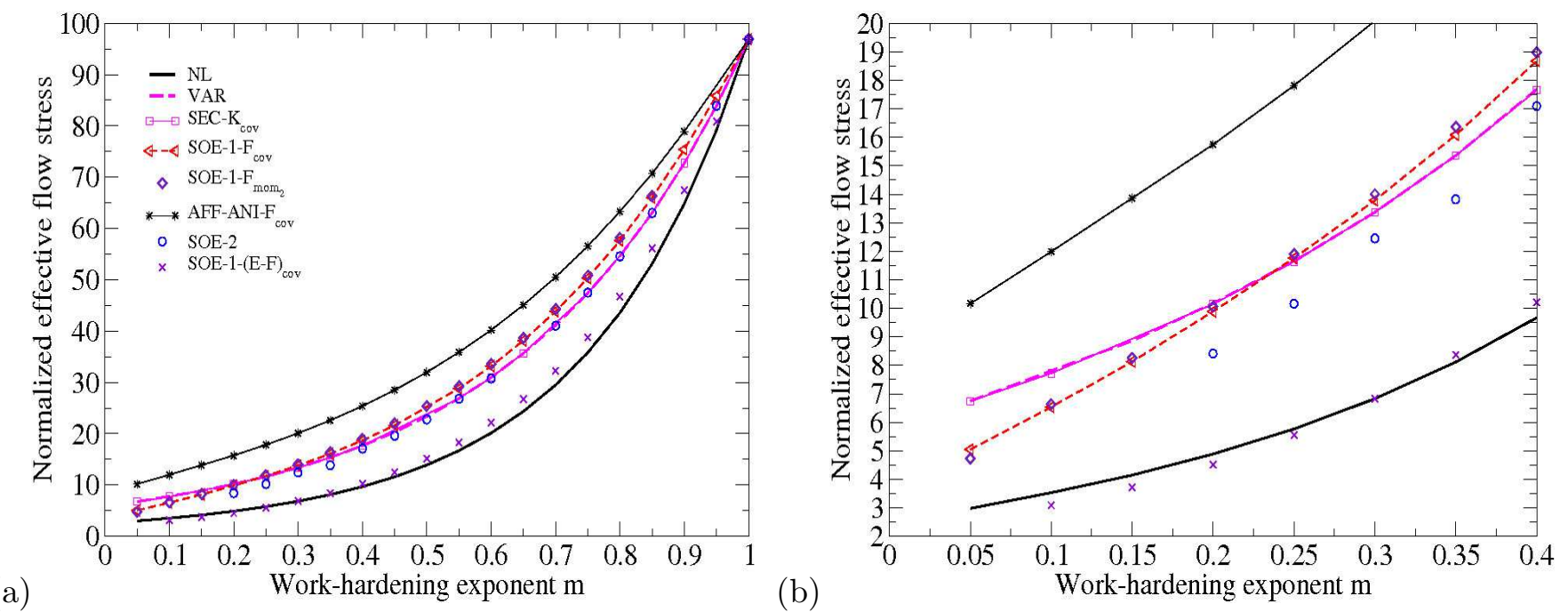

Figure 4: Porous media: variation of the normalized effective flow stress $\frac{\hat{\sigma}_{0}}{\sigma_{0}^{m}}$ associated with the linearization procedures $S E C$ $K_{\text {cov }}, A F F-A N I-F_{\text {cov }}, S O E-1-F_{\text {mom } 2}, S O E-1-F_{\text {cov }}$ and SOE-1-(E-F) cov with respect to the work-hardening exponent $m$. Results derived from VAR and SOE-2 linearization schemes defined in Sections B.1 and B.2 are also reported for comparison purposes. Full curves (a) and zoom (b). $f^{\mathrm{P}}=0.01$.

stresses which highly over-estimate the NL solution. This confirm again the necessity to account for both inter and intraphase strain fluctuations when linearizing the nonlinear behavior of phase $r$ as previously mentioned for instance in $[24,30]$. Figure (4) shows that, among all the proposed new formulations, only the enhanced tangent second order formulation SOE-1-(E-F)cov delivers good estimates for the whole range of nonlinearities including strong nonlinearities (i.e. when $m$ vanishes). Even though the SEC-K $\mathrm{K}_{\text {cov }}$ model, like the SOE-2 procedure, highly over-estimates the reference solution, it is consistent and even coincides with the rigorous variational bound VAR - since $\bar{\varepsilon}_{e q}^{r} \simeq 0$ and therefore $\hat{\varepsilon}_{e q}^{r} \simeq \bar{\varepsilon}_{e q}^{r}$ (see section (3.1) - unlike the

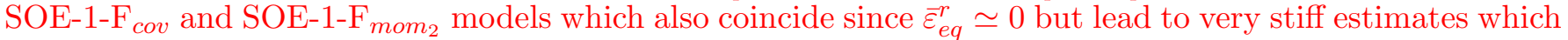
violate the VAR response for $m>0.3$. At last, even though the AFF-ANI-F cov model improves on the SEC, SOE-1, LS and existing affine formulations since it accounts for the intraphase strain fluctuations along the perpendicular direction $F^{r}$, it yields stiffer estimate than the enhanced secant SEC-K $\mathrm{K}_{\text {cov }}$ and second order SOE-1-F $\mathrm{F}_{\text {cov }}$, SOE-1-F mom $_{2}$ formulations. Accordingly it is not a satisfactory model when dealing with the prediction of the effective flow stress of porous materials under hydrostatic loading. This evaluation underline the fact that standard mean-field homogenization theories fail to reproduce the reference nonlinear solution of a porous media under hydrostatic loading except some rare and more sophisticated models like the ones developed by Bilger et al. [3, 4] or Danas et al. [9]. In the works of Bilger et al., a modified secant extension based on multilayered composite spheres - with a piecewise constant but non uniform distribution of shear moduli in the matrix - is presented for porous media with perfectly plastic matrix. This enriched model improves on the VAR estimate since it describes more accurately the local plastic flow or deformation gradient along the radii around the pores. Another relevant model for such situations was more recently designed by Danas et al. [9] in such a way that it reproduces exactly the behavior of a viscoplastic porous materials with a "composite-cylinder assemblage" microstructure in the limit of in-plane hydrostatic loading. The novel reference stress prescribed by this model depends on the stress triaxiality and the Lode angle [19] in stress space, the latter being related to the third invariant of the macroscopic stress tensor. This new reference strain allows to improve significantly on the earlier VAR and second-order estimates, especially at high stress triaxialities, low porosities and for high nonlinearities. 
The SOE-1-(E-F) cov model presented in this paper is shown in Fig. 4 to give estimates in good agreement with the reference solution for the whole range of nonlinearities in the limit of porous media for high triaxiality loading conditions. On the other hand, even though it improves on the estimates of the modified secant extensions (VAR and $\mathrm{SEC}_{\mathrm{K}} \mathrm{K}_{\text {cov }}$ ) and the second-order variants (SOE-1, SOE-2, SOE-1-F cov and SOE-1-F mom2 $_{2}$ ) under hydrostatic strain loading, it is expected to be too soft under isochoric extension and low triaxialities since, by construction, it is softer than the SOE-1-F $\mathrm{F}_{\text {cov }}$ approach which itself is softer, as observed in Figure 3, than the nonlinear solution for isochoric loadings. Accordingly, for such loadings, it is more appropriate to apply the SOE-1- $\mathrm{F}_{\text {cov }}$. Indeed, accounting for the intraphase strain fluctuations in the parallel direction $E^{r}$ added to the ones in the orthogonal direction, as it is the case of the SOE-1-(E-F) cov model, highly softens the effective flow stress of a porous media under pure isochoric extension and low strain triaxialities. However, as observed in the evolution of the SOE-1-(E-F) cov approach in Figure 4, this work shows that it is important to account for the intraphase field fluctuations in the parallel direction at intermediate and high triaxialities. Especially, for axisymmetric problems, the SOE-1-(E-F)cov could be a good alternative for second-order formulations (SOE-1, SOE-2) when dealing with porous media under moderate and high triaxilities.

Finally, it should be noted that the SOE-1-(E-F) cov approach is consistently defined even for the limit case of hydrostatic macroscopic strain loadings since, as defined by Eqs. $(8)_{4}$ and $(10)_{1}$, the second-order $e^{\mathrm{m}}=\hat{e}\left(\bar{\varepsilon}^{\mathrm{m}}\right)=e_{3} \otimes e_{3}-\frac{1}{2}\left(e_{1} \otimes e_{1}+e_{2} \otimes e_{2}\right)$ and fourth-order $E^{\mathrm{m}}=\frac{2}{3} e^{\mathrm{m}} \otimes e^{\mathrm{m}}$ tensors are well defined unlike $\hat{e}(\bar{\varepsilon})=\frac{\bar{\varepsilon}_{d}}{\bar{\varepsilon}_{e q}}$ which is undefined for hydrostatic macroscopic strains. The fact that $e^{\mathrm{m}}$ is equal to $e_{3} \otimes e_{3}-\frac{1}{2}\left(e_{1} \otimes e_{1}+e_{2} \otimes e_{2}\right)$, and therefore that $e^{\mathrm{m}}$ and $E^{\mathrm{m}}=\frac{2}{3} e^{\mathrm{m}} \otimes e^{\mathrm{m}}$ are still well defined for purely hydrostatic macroscopic strains (unlike $\hat{e}(\bar{\varepsilon})$ ), is due to the rotational invariance of the considered cylindrical unit cell along the third axis. Indeed, the latter implies the rotational invariance of the whole local problem since, as mentioned in Section 2.4, the behavior of the constituents and the loading are isotropic. Therefore, the deviatoric strain averages over the phases exhibit the same invariance and read $\bar{\varepsilon}_{d}^{r}=\hat{e} . \bar{\varepsilon}_{e q}^{r}$ with $\hat{e}=$ $e_{3} \otimes e_{3}-\frac{1}{2}\left(e_{1} \otimes e_{1}+e_{2} \otimes e_{2}\right)$. However, at this stage it should be emphasized that, for a porous medium with an isotropic matrix and now an isotropic (instead of transversely isotropic) microstructure (or unit cell) submitted to an hydrostatic macroscopic strain loading, there is no more any preferential anisotropic direction since the associated nonlinear local problem (Eq. (3)) is fully isotropic. By symmetry arguments, the strain averages over the phases are isotropic and their deviatoric parts are zero. Accordingly, the tensors $e^{\mathrm{m}}=\hat{e}\left(\bar{\varepsilon}^{\mathrm{m}}\right)$ and $E^{\mathrm{m}}$ are undefined and therefore the SOE-1-(E-F) cov approach as well. One exploratory way to overcome this difficulty might consist in introducing a preferential anisotropic direction $e^{\mathrm{m}}$ which would physically account for the anisotropy of the local strain field around the pores which actually occurs in real isotropic composites submitted to purely hydrostatic macroscopic loadings. Of course, the way to define such a preferential direction remains to be investigated. At last, note that the remarks formulated in this paragraph about the SOE-1-(E-F) cov are also valid for the SOE-1-F ${ }_{c o v}$ and SOE-1-F mom $_{2}$ formulations.

\section{Conclusions and perspectives}

Driven by some relevant results of non-biased comparative evaluations of various "stress-strain" or "potentialbased" linearization procedures and their interpretation, we have proposed five enhanced formulations derived from the earlier so-called secant, affine and tangent second-order linearization schemes. The key point relies on the replacements of the reference strain(s) used by these schemes in order to generate "softer" LCCs and better account for intraphase fluctuations of the strain field since it has been shown that the secant, classical affine and initial second-order formulations provide too stiff estimates. For the sake of simplicity and as a first illustration, the modified linearization schemes have been tested on both a periodic

rigidly-reinforced composite and a periodic porous media subjected to axisymmetric loading, making use 
of a non-biased evaluation procedure in which reference nonlinear composite and LCC exhibit the same microstructure and are numerically homogenized with an exact treatment.

A first conclusion of this objective evaluation is that the enhanced affine formulations, especially those incorporating more information about the intraphase strain fluctuations (i.e. AFF-ANI-F cov and AFFANI- $\mathrm{F}_{\text {mom }_{2}}$ ), even though more difficult to implement than the classical and simplified affine procedures, yield better results than earlier affine models. Such "stress-strain" formulations might be useful for "twopotential" materials for which other procedures such as the potential-based approaches are complex and difficult to implement.

With respect to the AFF-ANI-F ${ }_{\alpha}$ formulation, this parametric approach might appear attractive due to its simplicity and ease of use. However, it presents the trivial limitations not to account for intraphase fluctuations and to require a reference solution in order to fit the parameter $\alpha$. Accordingly, this formulation is more a phenomenological approach than a micromechanical one due to its fitting parameter $\alpha$ and should thus be used with much care. Indeed, the comparison of result of this model with reference solution in two different situations shows the limitation of such an empirical approach.

Our comparisons show also the limitations of secant linearizations. Our attempt to soften the modified secant (or variational) formulation, by the use of a larger reference strain, turned out to be unsuccessful. Even if the proposed SEC-K $\mathrm{K}_{\text {cov }}$ approach allowed to nicely capture the evolution of effective properties with nonlinearity exponent $m$ in some cases (for rigidly-reinforced composites at intermediate volume fractions), this approach was also found to be far too soft for porous media, and even to predict non physical results for rigidly reinforced composites at low volume fraction. This limitation seems to show that LCCs that depend on a single parameter are not sufficient to capture the complexity of local interactions in nonlinear media. Secant formulations might however be used as first approximations, because of their simpler implementations which sometimes even lead to analytical expressions, as shown in Appendix (C) where a new closed-form expression of the effective flow stress has been obtained for power-law porous materials.

In contrast, the SOE-1- $\mathrm{F}_{\text {mom }_{2}}$ and SOE-1- $\mathrm{F}_{\text {cov }}$ procedures showed their relevance to predict the overall behaviour for both the reinforced composites or porous media under low triaxiality loading conditions, even in sharp situations, i.e. for strong nonlinearity or near the percolation threshold. They yield very close estimates to the earlier second-order procedures SOE-2 which was known to be accurate and efficient. They even provides sometime more accurate predictions at least in terms of the macroscopic properties, see e.g. Fig (3). The SOE-1-F mom $_{2}$ procedure seems to be the more accurate and has the additional advantage to be easier to implement than SOE-1-F $\mathrm{F}_{\text {cov }}$.

As few existing nonlinear mean-field homogenization theories [3, 9], the SOE-1-(E-F) cov procedure is shown to provide good estimates for the effective flow stress on a very discriminant situation consisting of a porous medium under intermediate or high triaxiality loading conditions at low pore concentrations. However, this model yields (too) soft estimates at low triaxiality and its extension to purely isotropic structures under hydrostatic loadings is opened

Finally, it should be noted that all modified linearization procedures presented in this paper are derived by only changing the definitions of the tensors of elastic moduli of some of the (thermo)elastic LCCs defined in Sections B.1 and B.2 while letting their polarization tensors - when the LCC is thermoelastic unchanged. Another alternative, not treated in this paper, would consist to explore "new" polarization tensors for thermoelastic LCCs. Such an alternative is left or a future investigation. 


\section{A. Expressions of the stress field statistics}

\section{A.1. Definitions}

Similarly to the strain fluctuations (Eq. (14)), the parallel $\delta_{\|}^{r}(\sigma)$ and perpendicular $\delta_{\perp}^{r}(\sigma)$ measures of the fluctuations of the stress field in phase $r$ are defined by

$$
\delta_{\|}^{r}(\sigma)=\sigma_{\|}^{r}-\bar{\sigma}_{e q}^{r}=\sqrt{\frac{3}{2} E^{r}:: C_{\sigma}^{r}}, \quad \delta_{\perp}^{r}(\sigma)=\sigma_{\perp}^{r}=\sqrt{\frac{3}{2} F^{r}:: C_{\sigma}^{r}}
$$

with $\bar{\sigma}^{r}=\left\langle\sigma>^{r}\right.$ and $C_{\sigma}^{r}=\left\langle\left(\sigma-\bar{\sigma}^{r}\right) \otimes\left(\sigma-\bar{\sigma}^{r}\right)\right\rangle^{r}$. Note that the stress covariance tensor in phase $r$ also reads $C_{\sigma}^{r}=\langle\sigma \otimes \sigma\rangle^{r}-\bar{\sigma}^{r} \otimes \bar{\sigma}^{r}$ and therefore $\frac{3}{2} K:: C_{\sigma}^{r}=\left(\overline{\bar{\sigma}}_{e q}^{r}\right)^{2}-\left(\bar{\sigma}_{e q}^{r}\right)^{2}$ with $\overline{\bar{\sigma}}_{e q}^{r}=\sqrt{\left\langle\sigma_{e q}^{2}\right\rangle^{r}}$.

When the uniform stiffness tensor $L^{r}$ in phase $r$ is given by the general form $L^{r}=3 k^{r} J+2 \mu^{r} F^{r}+2 \lambda^{r} E^{r}$ - as it is the case of all the LCCs considered in this paper - the relationships between the measures of the stress and strain fluctuations in phase $r$ then read

$$
\delta_{\|}^{r}(\sigma)=3 \lambda^{r} \delta_{\|}^{r}(\varepsilon)=3 \lambda^{r}\left(\hat{\varepsilon}_{\|}^{r}-\bar{\varepsilon}_{e q}^{r}\right), \quad \delta_{\perp}^{r}(\sigma)=3 \mu^{r} \delta_{\perp}^{r}(\varepsilon)=3 \mu^{r} \hat{\varepsilon}_{\perp}^{r}
$$

since $C_{\sigma}^{r}=\left(L^{r} \otimes L^{r}\right):: C_{\varepsilon}^{r}$ for all (thermo)elastic linear constitutive behavior, i.e. $\sigma(x)=L^{r}: \varepsilon(x)\left(+\tau^{r}\right)$.

\section{A.2. Results}

We provide in this appendix additional results which complement those already published in [39] in order to sustain the discussion reported in Section 3.3. Fig.(5) depicts the evolutions with respect to the workhardening exponent $m$ of the parallel and orthogonal measures of the stress fluctuations over the matrix. Fig.(6) illustrates the evolutions of the parallel and orthogonal measures of the strain fluctuations over the matrix as functions of the inclusion volume fraction. The latter figure confirms that the predictions of the measures of the strain fluctuations over the matrix are very close for all the linearization schemes considered in this study.

(a) $\not$

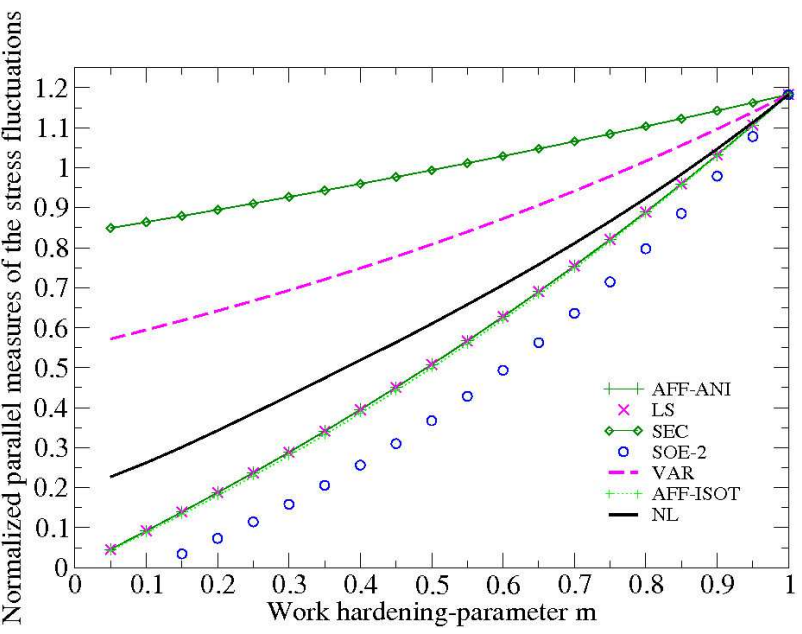

(b)

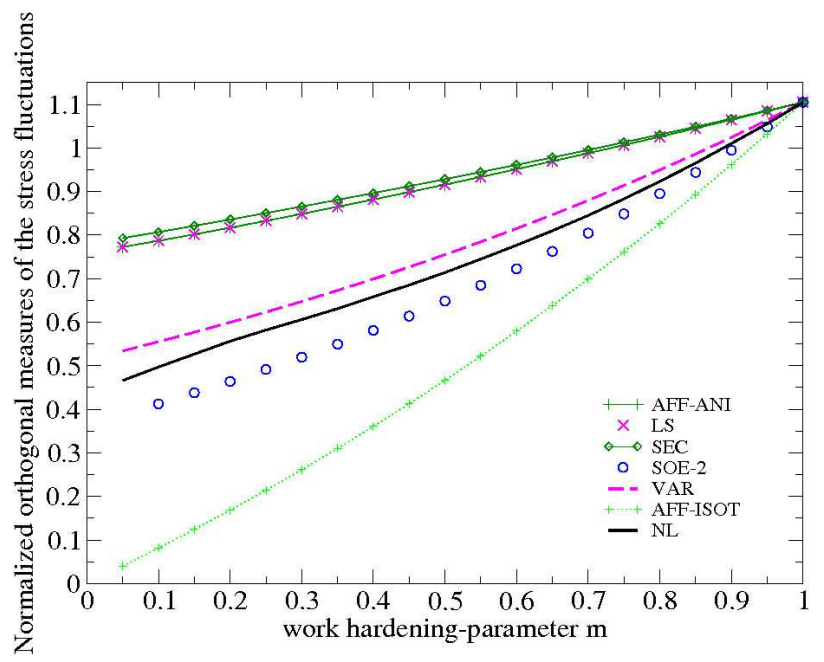

Figure 5: Reinforced composite: variation of (a) the normalized parallel $\frac{\hat{\sigma}_{\|}^{\mathrm{m}}-\bar{\sigma}_{e q}^{\mathrm{m}}}{\sigma_{0}^{\mathrm{m}}}$ and (b) orthogonal $\frac{\hat{\sigma}_{\frac{1}{\mathrm{~m}}}}{\sigma_{0}^{\mathrm{m}}}$ measures of the stress fluctuations over the matrix with respect to the work-hardening exponent $m . f^{\mathrm{p}}=0.3$. 
(a)

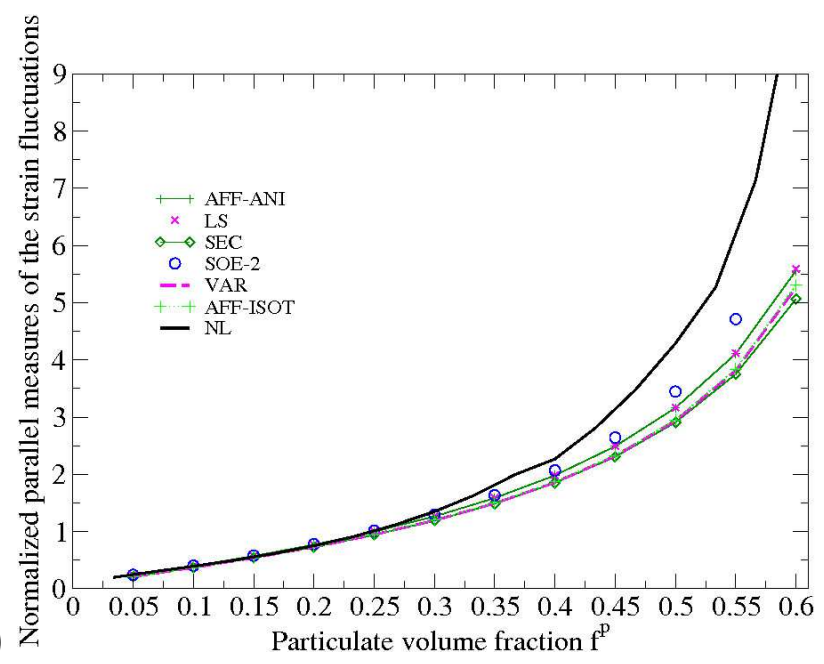

(b)

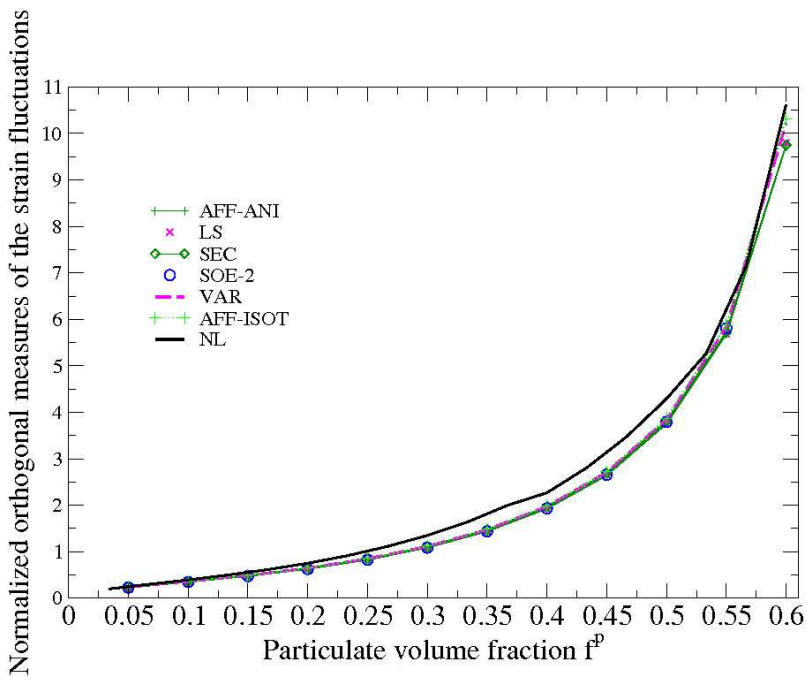

Figure 6: Reinforced composite: variation of (a) the normalized parallel $\frac{\hat{\varepsilon}_{\|}^{\mathrm{m}}-\bar{\varepsilon}_{e q}^{\mathrm{m}}}{\overline{\bar{\varepsilon}}_{e q}}$ and (b) orthogonal $\frac{\hat{\varepsilon}_{\perp}^{\mathrm{m}}}{\overline{\bar{\varepsilon}_{e q}}}$ measures of the strain fluctuations over the matrix with respect to the inclusion volume fraction $f^{\mathrm{p}} \cdot m=0.1$.

\section{B. Classical linearization procedures}

\section{B.1. Stress-strain approaches}

\section{B.1.1. Classical secant formulation (SEC)}

For this formulation, the LCC is elastic $\left(\tau^{r}=0\right)$. The local constitutive behaviour in phase $r$ of the LCC reads: $\sigma(x)=L^{r}: \varepsilon(x)$, where $L^{r}$ is defined as the isotropic tensor of secant moduli $(9)_{1}$ evaluated at a reference strain $\varepsilon^{r}$ defined as the equivalent phase average of the strain $\bar{\varepsilon}_{e q}^{r}$ in the LCC such that

$$
L^{r}=3 k^{r} J+2 \mu_{s c t}^{r}\left(\bar{\varepsilon}_{e q}^{r}\right) K .
$$

\section{B.1.2. Modified secant extension (VAR)}

This formulation proposed in [42] is identical to the classical secant formulation except for the fact that the reference strain $\varepsilon^{r}$ is now defined as the second-order moment of the strain field in phase $r \bar{\varepsilon}_{e q}^{r}$ such that

$$
L^{r}=3 k^{r} J+2 \mu_{s c t}^{r}\left(\overline{\bar{\varepsilon}}_{e q}^{r}\right) K
$$

The modified secant extension coincides with the variational approach of Ponte Castañeda [32].

\section{B.1.3. Classical affine formulation (AFF-ANI)}

In the original affine approach proposed by Masson et al [24], the LCC is thermo-elastic. Tensors $L^{r}$ and $\tau^{r}$ are respectively defined by $L^{r}=L_{t g t}^{r}\left(\varepsilon^{r}\right)=\frac{\partial^{2} w^{r}}{\partial \varepsilon^{2}}\left(\varepsilon^{r}\right)$ and $\tau^{r}=\frac{\partial w^{r}}{\partial \varepsilon}\left(\varepsilon^{r}\right)-L^{r}: \varepsilon^{r}$ for a reference strain $\varepsilon^{r}$ set to $\bar{\varepsilon}^{r}$. Unlike the secant methods, $L^{r}$ is anisotropic so that the tensors characterizing the LCC read

$$
L^{r}=L_{t g t}^{r}\left(\bar{\varepsilon}^{r}\right)=3 k^{r} J+2 \mu_{t g t}^{r}\left(\bar{\varepsilon}_{e q}^{r}\right) E^{r}+2 \mu_{s c t}^{r}\left(\bar{\varepsilon}_{e q}^{r}\right) F^{r}, \quad \tau^{r}=\frac{\partial w^{r}}{\partial \varepsilon}\left(\bar{\varepsilon}^{r}\right)-L^{r}: \bar{\varepsilon}^{r} .
$$




\section{B.1.4. Simplified affine formulation(AFF-ISOT)}

To simplify the original affine formulation which is anisotropic and to soften its prediction, Chaboche and Kanouté [8] have proposed a new variant referred to as AFF-ISOT with an isotropic tensor of elastic moduli defined by $L^{r}=3 k^{r} J+2 \mu_{t g t}^{r}\left(\varepsilon^{r}\right) K$ where $\varepsilon^{r}=\bar{\varepsilon}_{e q}^{r}$.

\section{B.2. Potential-based approaches}

\section{B.2.1. Second-order procedures: initial tangent formulation}

The initial second-order formulation also called the tangent second-order procedure and referred to in this study as SOE-1, is based on a second-order Taylor expansion [33] of the strain potential $w^{r}$ in each phase $r(r=1,2)$ around a reference strain $\varepsilon^{r}$ equal to the average strain in phase $\mathrm{r}$ within the LCC. While the derived LCC is exactly the same as the one of the affine formulation, the method to evaluate the effective response is different from the one associated with stress-strain approaches and relies on the construction of an effective potential estimated by

$$
\tilde{w}(\bar{\varepsilon}) \simeq \sum_{r} f^{r} w_{a p p}^{r}\left(\bar{\varepsilon}^{r}\right)=\sum_{r} f^{r}\left(w^{r}\left(\bar{\varepsilon}^{r}\right)+\frac{1}{2} \frac{\partial w^{r}}{\partial \varepsilon}\left(\bar{\varepsilon}^{r}\right):\left(\bar{\varepsilon}-\bar{\varepsilon}^{r}\right)\right),
$$

from which the effective stress is obtained according to $\bar{\sigma}=\frac{\partial \tilde{w}}{\partial \bar{\varepsilon}}(\bar{\varepsilon})$. The estimated local strain fields are the same in the affine and SOE-1 formulations unlike the per-phase stress averages which differ according to the chosen formulation (see [24]).

\section{B.2.2. Second-order procedures: improved versions}

For the improved second-order formulation SOE-2, based on stationarity conditions related to the evaluated effective energy [35], $L^{r}$ is defined by means of a generalized secant relation

$$
\frac{\partial w^{r}}{\partial \varepsilon}\left(\hat{\varepsilon}_{0}^{r}\right)-\frac{\partial w^{r}}{\partial \varepsilon}\left(\varepsilon^{r}\right)=L^{r}:\left(\hat{\varepsilon}_{0}^{r}-\varepsilon^{r}\right) .
$$

The first reference strain $\varepsilon^{r}$ is set to $\bar{\varepsilon}^{r}$. Under the simplifying assumption that the generalized secant tensor $L^{r}$ takes on the simple anisotropic form $L^{r}=3 k^{r} J+2 \mu^{r} F^{r}+2 \lambda^{r} E^{r}$, to satisfy the remnant stationarity conditions the additional reference strain $\hat{\varepsilon}_{0}^{r}$ is defined such that its "parallel" $\hat{\varepsilon}_{0 \|}^{r}=\left(\frac{2}{3} \hat{\varepsilon}_{0}^{r}: E^{r}: \hat{\varepsilon}_{0}^{r}\right)^{\frac{1}{2}}$ and "perpendicular" $\hat{\varepsilon}_{0 \perp}^{r}=\left(\frac{2}{3} \hat{\varepsilon}_{0}^{r}: F^{r}: \hat{\varepsilon}_{0}^{r}\right)^{\frac{1}{2}}$ components to $\hat{e}^{r}=\hat{e}\left(\bar{\varepsilon}^{r}\right)$, respectively satisfy the following relations $\hat{\varepsilon}_{0 \|}^{r}=\hat{\varepsilon}_{\|}^{r}$ and $\hat{\varepsilon}_{0 \perp}^{r}=\hat{\varepsilon}_{\perp}^{r}$ where the strain descriptors $\hat{\varepsilon}_{\|}^{r}, \hat{\varepsilon}_{\perp}^{r}$ have already been defined in Eq. (12). Finally, the effective stress is again obtained by means of Hill's theorem (1) for an effective potential approximated by $\tilde{w}(\bar{\varepsilon}) \simeq \sum_{r} f^{r} w_{a p p}^{r}\left(\bar{\varepsilon}^{r}, \hat{\varepsilon}_{0}^{r}\right)=\sum_{r} f^{r}\left(w^{r}\left(\hat{\varepsilon}_{0}^{r}\right)+\frac{1}{2} \frac{\partial w^{r}}{\partial \varepsilon}\left(\bar{\varepsilon}^{r}\right):\left(\bar{\varepsilon}^{r}-\hat{\varepsilon}_{0}^{r}\right)\right)$ where the strain field descriptors $\bar{\varepsilon}^{r}$ and $\hat{\varepsilon}_{0}^{r}$ (or equivalently $\hat{\varepsilon}_{\|}^{r}, \hat{\varepsilon}_{\perp}^{r}$ ) are derived from the LCC ${ }^{2}$. Note that a third version of the second order procedure SOE-3, very similar to the SOE-2 version, is also proposed in [14, 17]. For the sake of simplicity, we will only consider the SOE-1 and SOE-2 second-order procedures based on a strain potential $w(\varepsilon)$.

\footnotetext{
${ }^{2}$ To derive closed-form expression of $w_{a p p}^{r}$ as functions of $\hat{\varepsilon}_{\|}^{r}, \hat{\varepsilon}_{\perp}^{r}$ (and not of $\hat{\varepsilon}_{0}^{r}$ ), more information about the phase constitutive laws should be available. See for instance [36] where closed-form expressions of $w_{a p p}^{r}\left(\bar{\varepsilon}^{r}, \hat{\varepsilon}_{\|}^{r}, \hat{\varepsilon}_{\perp}^{r}\right)$ are given for rigidly reinforced composites and porous materials
} 


\section{B.2.3. The Lahellec and Suquet scheme (LS)}

The Lahellec and Suquet formulation LS [21] retains the energetic framework of the tangent second-order formulation and modifies it in such a way that the "stress-strain" formulation $(\bar{\sigma}=\langle\sigma\rangle)$ is in exact agreement with the potential-based formulation $\left(\bar{\sigma}=\frac{\partial \tilde{w}}{\partial \bar{\varepsilon}}(\bar{\varepsilon})\right)$. The following expressions are generated for the constitutive relations of the LCC

$$
L^{r}=L_{t g t}^{r}\left(\bar{\varepsilon}^{r}\right), \quad \tau^{r}=\frac{\partial w^{r}}{\partial \varepsilon}\left(\bar{\varepsilon}^{r}\right)-L^{r}: \bar{\varepsilon}^{r}+\frac{1}{2} N^{r}\left(\bar{\varepsilon}^{r}\right):: C_{\varepsilon}^{r}, \quad \text { with } \quad N^{r}(\varepsilon)=\frac{\partial^{3} w^{r}}{\partial \varepsilon^{3}}(\varepsilon) .
$$

Calculations made in [38] show that $N^{r}\left(\bar{\varepsilon}^{r}\right):: C_{\varepsilon}^{r}=\left(2 \frac{d \mu_{t g t}^{r}\left(\bar{\varepsilon}_{e q}^{r}\right)}{d \varepsilon_{e q}}\left(\hat{\varepsilon}_{\|}^{r}-\bar{\varepsilon}_{e q}^{r}\right)^{2}+\frac{4}{3} \frac{d \mu_{s c t}^{r}\left(\bar{\varepsilon}_{e q}^{r}\right)}{d \varepsilon_{e q}}\left(\hat{\varepsilon}_{\perp}^{r}\right)^{2}\right) \hat{e}^{r}$, where $\hat{\varepsilon}_{\|}^{r}$, $\hat{\varepsilon}_{\perp}^{r}$ are defined by Eq. (12) such that $L^{r}$ and $\tau^{r}$ only depend on three statistical descriptors $\bar{\varepsilon}^{r}, \hat{\varepsilon}_{\|}^{r}, \hat{\varepsilon}_{\perp}^{r}$ of the local strain field within the LCC. Finally, the effective strain-potential is approximated by $\tilde{w}(\bar{\varepsilon}) \simeq$ $\sum_{r} f^{r}\left(w^{r}\left(\bar{\varepsilon}^{r}\right)+\frac{1}{2} \frac{\partial w^{r}}{\partial \varepsilon}\left(\bar{\varepsilon}^{r}\right):: C_{\varepsilon}^{r}\right)$ and the macroscopic stress $\bar{\sigma}$ might be evaluated either as the average value of the local stress field within the LCC or by means of Hill's theorem through Eq. (2) 1 .

\section{Additional analytical result regarding secant linearization schemes (SEC, VAR, SEC-K cov $_{\text {) }}$ for power-law porous materials}

This appendix provides an analytical proof of the almost affine evolution of the effective flow stress $\tilde{\sigma}_{0}$ with respect to the work-hardening exponent $m$, for power-law porous material under pure deviatoric extension, observed in Fig. 3.

The proposed proof applies even to a somewhat more general situation: we consider a porous material with an isotropic incompressible nonlinear elastic matrix, with a nonlinear behavior characterized by the work-hardening curve $\sigma_{e q}=\sigma^{\mathrm{m}}\left(\varepsilon_{e q}\right)$, from which the secant modulus $\mu_{s c t}^{\mathrm{m}}\left(\varepsilon_{e q}\right)=\frac{\sigma^{\mathrm{m}}\left(\varepsilon_{e q}\right)}{3 \varepsilon_{e q}}$ can be defined. The matrix is assumed insensitive to the third invariant of the strain. This porous material is subjected to an overall strain $\bar{\varepsilon}$, with deviatoric part $\bar{\varepsilon}_{d}$, von Mises equivalent strain $\bar{\varepsilon}_{e q}$, triviality ratio $\bar{\tau}_{\varepsilon}$ and direction $\hat{e}=\hat{e}(\bar{\varepsilon})$.

A special case would be a power-law type behavior, such that

$$
\mu_{s c t}^{\mathrm{m}}\left(\varepsilon_{e q}\right)=\frac{\sigma_{0}}{3 \varepsilon_{0}}\left(\frac{\varepsilon_{e q}}{\varepsilon_{0}}\right)^{m-1} .
$$

The microstructure of the porous material does not need to be specified: the result is not restricted to the particular geometry considered throughout the paper.

When a secant linearization scheme is considered, the LCC is made of the same arbitrary microgeometry and is subjected to the same overall strain $\bar{\varepsilon}$. The linear behavior of the matrix in the LCC depends on the sole shear modulus $\mu^{\mathrm{m}}$, which takes various values depending on which secant scheme is considered. The essential property on which the final result relies on, is however that the local strain field in the LCC, for a fixed overall prescribed strain $\bar{\varepsilon}$, does not depend on the value of this shear modulus $\mu^{m}$. We refer to Appendix D of [40] for a straightforward proof of this property. Since the localization problem in the LCC is linear, one can classically define a fourth order localization tensor $A^{\mathrm{m}}$ relative the the matrix such that $\bar{\varepsilon}^{\mathrm{m}}=A^{\mathrm{m}}: \bar{\varepsilon}$, which does not depend on $\mu^{\mathrm{m}}$. In addition, one can define the following ratios, which do not depend on $\mu^{\mathrm{m}}$ either

$$
\gamma\left(\bar{\tau}_{\varepsilon}, \hat{e}\right)=\frac{\bar{\varepsilon}_{e q}^{\mathrm{m}}}{\bar{\varepsilon}_{e q}} \quad \text { and } \quad \gamma^{r}\left(\bar{\tau}_{\varepsilon}, \hat{e}\right)=\frac{\varepsilon^{r}}{\bar{\varepsilon}_{e q}}
$$


where $\varepsilon^{r}$ is the scalar reference strain in phase $r$ used to compute the secant modulus, and defined, for the three considered secant schemes, by:

$$
\varepsilon^{r}=\bar{\varepsilon}_{e q}^{r}(\mathrm{SEC}), \quad \bar{\varepsilon}_{e q}^{r}(\mathrm{VAR}) \quad \text { or } \quad \hat{\varepsilon}_{e q}^{r}\left(\mathrm{SEC}-\mathrm{K}_{\text {cov }}\right) .
$$

For all these secant schemes, the overall deviatoric stress in the nonlinear porous material is obtained by averaging the stress in the LCC:

$$
\bar{\sigma}_{d}=\left(1-f^{\mathrm{p}}\right) \bar{\sigma}_{d}^{\mathrm{m}}=2\left(1-f^{\mathrm{p}}\right) \mu_{s c t}^{\mathrm{m}}\left(\varepsilon^{\mathrm{m}}\right) \bar{\varepsilon}_{d}^{\mathrm{m}}=2\left(1-f^{\mathrm{p}}\right) \mu_{s c t}^{\mathrm{m}}\left(\gamma^{r}\left(\bar{\tau}_{\varepsilon}, \hat{e}\right) \bar{\varepsilon}_{e q}\right) K:\left(A^{\mathrm{m}}: \bar{\varepsilon}\right)
$$

so that in particular the von Mises equivalent macroscopic stress reads:

$$
\bar{\sigma}_{e q}=3\left(1-f^{\mathrm{p}}\right) \mu_{s c t}^{\mathrm{m}}\left(\gamma^{r}\left(\bar{\tau}_{\varepsilon}, \hat{e}\right) \bar{\varepsilon}_{e q}\right) \gamma\left(\bar{\tau}_{\varepsilon}, \hat{e}\right) \bar{\varepsilon}_{e q}
$$

This expression provides a way to construct analytically the secant nonlinear extension of a linear model for any porous material with an incompressible isotropic matrix: one has just to compute the expressions of $A^{\mathrm{m}}, \gamma^{r}$ and $\gamma$ for the considered overall load $\bar{\varepsilon}$, from the linear model defined for an arbitrary value of $\mu^{\mathrm{m}}$.

Now for the special case of a power-law matrix, it is well known [34] that the effective behavior follows a power-law with same exponent

$$
\bar{\sigma}_{e q}=\tilde{\sigma}_{0}\left(\bar{\tau}_{\varepsilon}, \hat{e}\right)\left(\frac{\bar{\varepsilon}_{e q}}{\varepsilon_{0}}\right)^{m}
$$

This result is also a direct consequence of the homogeneity of degree one of the dependence of the local strain fields in the porous material with respect to the macroscopic strain.

Putting together equations (39), (37) and (34), one finally obtains

$$
\tilde{\sigma}_{0}\left(\bar{\tau}_{\varepsilon}, \hat{e}\right)=\left(1-f^{\mathrm{p}}\right) \gamma\left(\bar{\tau}_{\varepsilon}, \hat{e}\right) \sigma_{0}\left(\gamma^{\mathrm{m}}\left(\bar{\tau}_{\varepsilon}, \hat{e}\right)\right)^{m-1} .
$$

For the situation considered in Fig. 3, the triaxiality ratio $\bar{\tau}_{\varepsilon}$ is null and the overall load is always proportional to $\hat{e}=e_{3} \otimes e_{3}-\frac{1}{2}\left(e_{1} \otimes e_{1}+e_{2} \otimes e_{2}\right)$. However, strictly speaking, in Fig. 3 , the matrix is not incompressible but follows a Ramberg-Osgood relation $(15)_{2}$ with a finite initial elastic shear modulus and a finite elastic bulk modulus. But when the elastic part of the total strain in the matrix is negligible - i.e. for sufficiently large strain - which is actually the case in our study since the macroscopic constitutive law (39) of the reinforced composite is evaluated from the asymptotic behavior for large strains, the previous result for purely incompressible power law matrix almost holds true. Accordingly, the effective flow stress provided by a secant linearization and its derivative with respect to the work-hardening exponent $m$ read

$$
\tilde{\sigma}_{0} \approx\left(1-f^{\mathrm{p}}\right) \gamma \sigma_{0}\left(\gamma^{\mathrm{m}}\right)^{m-1}, \quad \frac{\partial \tilde{\sigma}_{0}}{\partial m} \approx \tilde{\sigma}_{0} \ln \left(\gamma^{\mathrm{m}}\right)=\underbrace{\left(1-f^{\mathrm{p}}\right) \gamma \sigma_{0} \ln \left(\gamma^{\mathrm{m}}\right)}_{\text {does not depend on } m}\left(\gamma^{\mathrm{m}}\right)^{m-1} .
$$

The slope $\frac{\partial \tilde{\sigma}_{0}}{\partial m}$ is almost independent on the exponent $m$, as $\tilde{\sigma}_{0}$ does not deviate strongly from its value for $m=1$, as observed in Fig. 3. This results from the fact that $\gamma^{\mathrm{m}}$ is never significantly different from 1 . As a consequence one observes a quasi-affine dependance with $m$ of the effective flow stress predicted by all secant models.

The slope is however strongly sensitive to the considered secant extension, through the value of $\gamma^{\mathrm{m}}$ which depends only on the linear local problem. Indeed, if $\gamma^{\mathrm{m}}<1$ then $\frac{\partial \tilde{\sigma}_{0}}{\partial m}<0$ and $\tilde{\sigma}_{0}$ decreases with increasing values of $m$ as observed for the SEC and VAR overall responses (see Fig. 3). If $\gamma^{\mathrm{m}}>1$ then $\frac{\partial \tilde{\sigma}_{0}}{\partial m}>0$ and $\tilde{\sigma}_{0}$ increases with $m$ as observed for the SEC-K $\mathrm{K}_{\text {cov }}$ overall predictions on Fig. 3. The slope is essentially related to whether the reference strain is larger or not than the overall equivalent strain. 


\section{References}

[1] Abdul-Latif, A., Dingli, J.Ph., Saanouni, K., 1998. Modeling of complex cyclic inelasticity in heterogeneous polycrystalline microstructure. Mechanics of Materials, 30, 287-305.

[2] Berveiller, M., Zaoui, A., 1979. An extension of the self-consistent scheme to plastically-following polycrystals. J. Mech. Phys. Solids 26, 325-344.

[3] Bilger, N., Auslender, F., Bornert, M., Masson, R., 2002. New bounds and estimates for porous media with rigid perfectly plastic. C. R. Mécanique 330, 127-132.

[4] Bilger, N., Auslender, F., Bornert, M., Moulinec, H., Zaoui, A., 2007. Bounds and estimates for the effective yield surface of porous media with a uniform or a nonuniform distribution of voids. Eur. J. of Mech. A/Solids, 26, 810-836.

[5] Bornert, M., Ponte Castañeda, P., 1998. Second-order estimates of the self-consistent type for viscoplastic polycrystals. Proc. R. Soc. Lond., A356, 3035-3045.

[6] Brenner, R., Castelnau, O., Gilormini, P., 2001. A modified affine theory for the overall properties of nonlinear composites. C. R. Acad. Sci. Paris, t. 329, Série IIb, 649-654.

[7] Cast3M official website: http://www-cast3m.cea.fr/cast3m/index.jsp

[8] Chaboche, J.L., Kanouté, P., 2003. Sur les approximations "isotrope" et "anisotrope" de l'opérateur tangent pour les méthodes tangentes incrémentale et affine. C. R. Mécanique 331, 857-864.

[9] Danas, K., Idiart, M.I., Ponte Castañeda, P., 2008. A homogenization-based constitutive model for two-dimensional viscoplastic porous media. C. R. Mécanique, 336, 79-90.

[10] Gilormini, P., 1995. Insuffisance de l'extension classique du modéle auto-cohérent au comportement non linéaire. C. R. Acad. Sci. Paris 320 (Série IIb), 115-122.

[11] Gilormini, P. , Brenner, R., Castelnau, O., 2001. A similarity between the classical and modified secant extensions of the self-consistent model, C. R. Mécanique 329, 523-527.

[12] Gilormini, P., Nebozhyn, M.V., Ponte Castañeda, P., 2001. Accurate estimates for the creep behavior of hexagonal polycrystals. Acta Materialia, 49 (2001), 329-337.

[13] Hill, R., 1967. The essential structure of constitutive laws for metal composites and polycrystals. J. Mech. Phys. Solids, 15, 79-95.

[14] Idiart, M.I., Ponte Castañeda, P., 2005. Second-order estimates for nonlinear isotropic composites with spherical pores and rigid particles. C. R. Mécanique, 333, 147-154.

[15] Idiart, M.I., Ponte Castañeda, P., 2003. Field fluctuations and macroscopic properties for nonlinear composites, Int. J. Solids Struct., 40, 7015-7033.

[16] Idiart, M.I., Moulinec, H., Ponte Castañeda, P., Suquet, P., 2006. Macroscopic behavior and field fluctuations in viscoplastic composites: second-order estimates vs full-field simulations. J. Mech. Phys. Solids 54, 1029-1063.

[17] Idiart, M.I., Ponte Castañeda, P., 2007. Field statistics in nonlinear composites. I. Theory, Proc. R. Soc. Lond. A463, 183-202. 
[18] Idiart, M.I., Danas, K., Ponte Castañeda, P., 2006. Second-order theory for nonlinear composites and application to isotropic constituents. C. R. Mécanique 334, 575-581.

[19] Kachanov, L.M., 1971. Foundations of the Theory of Plasticity. North-Holland, Amsterdam.

[20] Koplik, J., Needleman, A., 1988. Void growth and coalescence in porous plastic solids. Int. J. Solids Stuctures, 24, 835-853.

[21] Lahellec, N., Suquet, P., 2004. Nonlinear composites: a linearization procedure, exact to second-order in contrast and for which the strain-energy and affine formulations coincide. C. R. Mécanique 332, 693-700.

[22] Leblond, J.B., Perrin, G., Suquet, P., 1994. Exact results and approximate models for porous viscoplastic solids. Int. J. Plasticity 10, 213-235.

[23] Leroy, Y., Ponte Castañeda, P., 2001. Bounds on the self-consistent approximation for nonlinear media and implications for the second-order method. C. R. Mécanique 329, 571-577.

[24] Masson, R., Bornert, M., Suquet, P., Zaoui, A., 2000. An affine formulation for the prediction of the effective properties of nonlinear composites and polycrystals. J. Mech. Phys. Solids 48, 1203-1227.

[25] Michel, J.C., Moulinec, H., Suquet, P., 1999. Effective properties of composite materials with periodic microstructure: a computational approach. Computer Methods in Applied Mechanics and Engineering, $172(1-4), 109-143$.

[26] Molinari, A., Ahzi, S., Kouddane, R., 1997. On the self-consistent modeling of elastic-plastic behavior of polycrystals. Mechanics of Materials, 26, 43-62.

[27] Molinari, A., Tóth, L.S., 1994. Tuning a self-consistent visco-plastic model by finite element results, part I: modelling. Acta Metall. Mater., 42, 2453-2458.

[28] Moulinec, H., Suquet, P., 2003. Intraphase strain heterogeneity in nonlinear composites: a computational approach. Eur. J. of Mech. A/Solids 22, 751-770.

[29] Moulinec, H., Suquet, P., 2004. Homogenization for nonlinear composites in the light of numerical simulations. In Ponte Castañeda P., Telega J.J. (Eds), Nonlinear Homogenization and Its Applications to Composites, Polycrystals and Smart Materials. Kluwer Academic Publishers, The Netherlands, 193223.

[30] Pastor, J., Ponte Castañeda, P., 2002. Yield criteria for porous media in plane strain: second-order estimates versus numerical results. C. R. Mécanique, 330 (11), 741-747.

[31] Perrin, G., Leblond, J.B., 1990. Analytical study of a hollow sphere made of plastic porous material and subjected to hydrostatic tension. Application to some problems in ductile fracture of metals. Int. J. Plasticity 6, 677-699

[32] Ponte Castañeda, P., 1991. The effective mechanical properties of nonlinear isotropic composites. J. Mech. Phys. Solids, 39, 45-71.

[33] Ponte Castañeda, P., 1996. Exact second-order estimates for the effective mechanical properties of nonlinear composite materials. J. Mech. Phys. Solids 44, 827-862. 
[34] Ponte Castañeda, P., Suquet, P., 1998. Nonlinear composites. Advances in Applied Mechanics, Academic Press 34, 171-302.

[35] Ponte Castañeda, P., 2002. Second-order homogenization estimates for nonlinear composites incroporating field fluctuations: I- Theory. J. Mech. Phys. Solids 50, 737-757.

[36] Ponte Castañeda, P., 2002. Second-order homogenization estimates for nonlinear composites incroporating field fluctuations: II- Applications. J. Mech. Phys. Solids 50, 759-782.

[37] Rekik, A., Bornert, M., Auslender, F., Zaoui, A., 2005. A methodology for an accurate evaluation of the linearization procedures in nonlinear mean-field homogenization. C. R. Mécanique, 333 (11), 789-795.

[38] Rekik, A., 2006. Une méthodologie pour une évaluation précise des procédures de linéarisation en homogénéisation non linéaire, Ph.D. thesis, École Polytechnique.

[39] Rekik, A., Auslender, F., Bornert, M., Zaoui, A., 2007. Objective evaluation of linearization procedures in nonlinear homogenization: A methodology and some implications on the accuracy of micromechanical schemes. Int. J. Solids and Struct., 44 (10), 3468-3496.

[40] Rekik, A., Bornert, M., Auslender, F., 2012. A critical evaluation of local field statistics predicted by various linearization schemes in nonlinear mean-field homogenization. Mechanics of Materials, 54, 1-17.

[41] Segurado, J., Llorca, J., González, C., 2002. On the accuracy of mean-field approaches to simulate the plastic deformation of composites. Scripta Materliallia, 46 (7), 525-529.

[42] Suquet, P., 1995. Overall properties of nonlinear composites: a modified secant moduli theory and its link with Ponte Castañeda's nonlinear variational procedure. C. R. Mécanique, 320, 563-571.

[43] Tvergaard, V., 1982. On localization in ductile materials containing spherical voids. Int. J. Fracture, $18,237-252$. 